\title{
Biased Nearest Neighbour Search
}

\author{
By \\ Daniel Minor \\ A thesis submitted to \\ the Faculty of Graduate Studies and Research \\ in partial fulfilment of \\ the requirements for the degree of \\ Master of Computer Science \\ Ottawa-Carleton Institute for Computer Science \\ School of Computer Science \\ Carleton University \\ Ottawa, Ontario
}

December 2014

(C) Copyright

2014, Daniel Minor 
The undersigned hereby recommend to the Faculty of Graduate Studies and Research aceeptance of the thesis,

\section{Biased Nearest Neighbour Search}

submitted by

Daniel Minor

Douglas Howe

(Director, School of Computer Science)

Patrick Morin

(Thesis Supervisor)

Carleton University

December 2014 


\section{Abstract}

The nearest neighbour search problem is to preprocess a set of points so that for any query point, the closest point in the set to that query point can be determined quickly. In the biased version of this problem a probability distribution over the search points is available that can be used to answer searches more efficiently on average. This thesis contains empirical results showing that practical implementations of data structures for the biased nearest neighbour search problem are possible. 


\section{Acknowledgements}

I would like to thank my supervisor Dr. Pat Morin for his willingness to take on a part-time student and his advice and patience over the past four years.

Thank you April, Deirdre and Tamsin for your love and support.

To the staff of the Bridgehead at Golden Avenue in memory of many an early Sunday morning spent in one another's company. 


\section{Contents}

1 Introduction 1

1.1 Problem Statement . . . . . . . . . . . . . . . . . . . . . 1

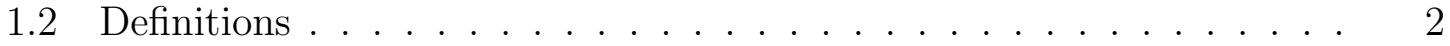

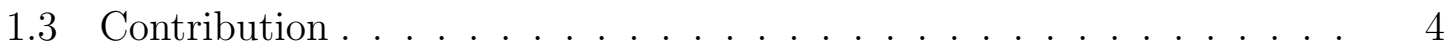

1.4 Organization of the Thesis $\ldots \ldots \ldots \ldots \ldots \ldots$

2 Previous Work $\quad 5$

2.1 Nearest Neighbour Search . . . . . . . . . . . . . . . 5

2.1.1 General Algorithm for Nearest Neighbour Search . . . . . . 7

2.1 .2 Voronoi Diagrams . . . . . . . . . . . . . . . . . 10

2.1 .3 Quadtrees ........................ 11

2.1.4 Compressed Quadtrees . . . . . . . . . . . . . . . 13

2.1.5 Skip Quadtrees . . . . . . . . . . . . . . . . . 16

$2.1 .6 \quad$ Kd-Trees . . . . . . . . . . . . . . . . . . . . . . 17

2.1.7 Balanced Box Decomposition (BBD) Trees . . . . . . . . . . 19

2.1.8 Approximate Voronoi Diagrams . . . . . . . . . . . . . . 21

2.2 Biased Search . . . . . . . . . . . . . . . . . . . . . . 22

2.2.1 Information Theory . . . . . . . . . . . . . . . . . . 22

2.2 .2 Biased Search Trees . . . . . . . . . . . . . . . . . . 23

2.2 .3 Splay Trees . . . . . . . . . . . . . . . . . . 25

2.2.4 Planar Point Location _ . . . . . . . . . . . . . . . . 26

2.2 .5 Biased Range Trees . . . . . . . . . . . . . . . . . . . 27

2.2 .6 Odds-on Trees . . . . . . . . . . . . . . . . . . . . 30 
3 Implementation 33

3.1 Kd-tree Implementation . . . . . . . . . . . . . . . . . . . . . 34

3.2 Kd-Tree Based Odds-on Tree Implementation . . . . . . . . . . . . . 35

3.3 Quadtree Based Odds-on Tree Implementation . . . . . . . . . . . . . 36

3.4 Test Harness Implementation _. . . . . . . . . . . . . . . . . 37

4 Experimental Results $\quad 38$

4.1 Experiment Design . . . . . . . . . . . . . . . . . 38

4.1.1 Dimension . . . . . . . . . . . . . . . . . 38

4.1 .2 Point Set Size . . . . . . . . . . . . . . . . . . . 39

4.1.3 Search Set Entropy . . . . . . . . . . . . . . . . 39

4.1.4 Sample Set Size . . . . . . . . . . . . . . . . . . . . . 42

4.1.5 Maximum Build Depth . . . . . . . . . . . . . . . . . . 42

4.1.6 Number of Runs . . . . . . . . . . . . . . . . . . . . 43

4.2 Measuring Performance . . . . . . . . . . . . . . 43

4.2.1 Benchmark Platform ............... . . 43

4.2.2 Performance Metric . . . . . . . . . . . . . . . . . 43

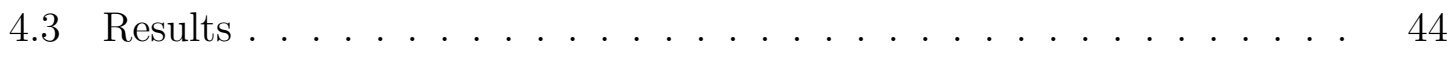

4.3.1 Dimension ....................... 44

4.3 .2 Point Set Size . . . . . . . . . . . . . . . . . 44

4.3 .3 Search Set Entropy . . . . . . . . . . . . . . . . 48

4.3 .4 Sample Set Size . . . . . . . . . . . . . . . . . . . 49

4.3.5 Maximum Build Depth . . . . . . . . . . . . 52

4.3.6 Quadtree vs. Kd-Tree Based Implementation . . . . . . . . . . 55

5 Conclusions $\quad 61$

5.1 Summary ............................. 61

5.2 Future Work . . . . . . . . . . . . . . . . . 62

$\begin{array}{lr}\text { Bibliography } & 64\end{array}$ 


\section{List of Figures}

2.1 Representing Nearest Neighbour Search with Axis Aligned Hypercubes 6

2.2 Nearest Neighbour Search Algorithm . . . . . . . . . . . . . . . 9

2.3 Voronoi Diagram . . . . . . . . . . . . . . . . . . . . . . . . 10

2.4 Quadtree . . . . . . . . . . . . . . . . . . . . . . 12

2.5 Z-Order . . . . . . . . . . . . . . . . . . . . . . . . 14

2.6 Compressed Quadtree . . . . . . . . . . . . . . . . . 15

2.7 Kd-Tree . . . . . . . . . . . . . . . . . . . . . . . . . . . . . . . . 18

2.8 Balanced Box Decomposition Tree Nodes . . . . . . . . . . . . . 20

2.9 The range counting lower bound is not always achievable . . . . . . 29

4.1 Points and sample distributions $(2 \mathrm{~d}$, sigma $=0.01) \ldots \ldots \ldots$

4.2 Points and sample distributions $(2 \mathrm{~d}$, sigma $=0.05) \ldots \ldots \ldots$

4.3 Points and sample distributions $(2 \mathrm{~d}$, sigma $=0.10) \ldots \ldots \ldots 41$

4.4 Points and sample distributions $(2 \mathrm{~d}$, sigma $=0.25) \ldots \ldots \ldots 41$

4.5 Points and sample distributions $(2 \mathrm{~d}$, sigma $=0.50) \ldots \ldots \ldots . \quad \ldots 2$

4.6 Query Time $(\mathrm{pts}=50000$, sample $=25000$, sigma $=0.01) \ldots \ldots \quad 45$

4.7 Construction Time $(\mathrm{kd}-\mathrm{tree}, 2 \mathrm{~d}$, sigma $=0.01) \ldots \ldots \ldots \ldots$

4.8 Query Time $(\mathrm{kd}$-tree, $2 \mathrm{~d}$, sigma $=0.01) \ldots \ldots \ldots \ldots$

4.9 Construction Time (quadtree, $2 \mathrm{~d}$, sigma $=0.01) \ldots \ldots \ldots . \quad \ldots$

4.10 Query Time (quadtree, $2 \mathrm{~d}$, sigma $=0.01) \ldots \ldots \ldots \ldots . \ldots 47$

4.11 Query Time (kd-tree, $2 \mathrm{~d}$, pts $=10000$, sample=5000) . . . . . . 48

4.12 Construction Time (kd-tree, 2d, pts=10000, sample=5000) … . 49

4.13 Node Counts (kd-tree, 2d, pts $=10000$, sample $=10000$, build depth=13) 50

4.14 Construction Time $(k d-t r e e, 2 \mathrm{~d}, \mathrm{pts}=10000) \ldots \ldots \ldots \ldots \ldots$ 
4.15 Query Time(kd-tree, 2d, pts=10000) . . . . . . . . . . . . 51

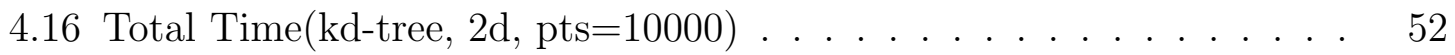

4.17 Node Counts (kd-tree, 2d, pts=10000) . . . . . . . . . . . 53

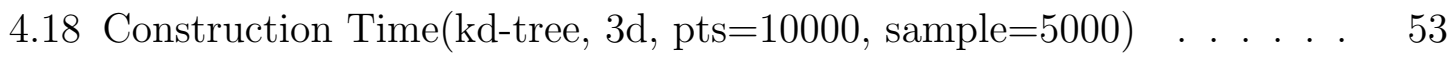

4.19 Query Time $(k d-t r e e, 3 d, p t s=10000$, sample=5000) . . . . . . . . . 54

4.20 Total Time $(k d-t r e e, 3 d, p t s=10000$, sample=5000) . . . . . . . . . . 54

4.21 Construction Time(quadtree, $3 \mathrm{~d}, \mathrm{pts}=10000$, sample=5000) . . . . . 55

4.22 Query Time(quadtree, 3d, pts $=10000$, sample=5000) . . . . . . . 56

4.23 Build Time(3d, pts=10000) . . . . . . . . . . . . . 57

4.24 Query Time $(3 \mathrm{~d}, \mathrm{pts}=10000) \ldots \ldots \ldots$. . . . . . . . . 57

4.25 Total Time $(3 \mathrm{~d}, \mathrm{pts}=10000) \ldots \ldots \ldots$. . . . . . . . . . 58

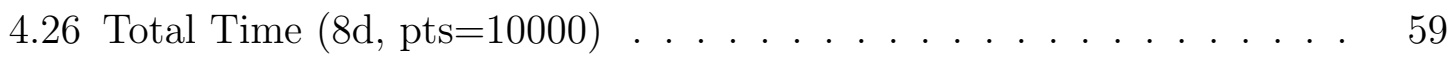

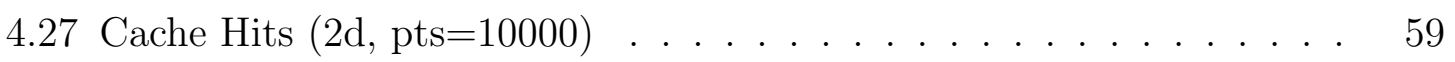

4.28 Square vs Rectangular Nodes . . . . . . . . . . . . . . . 60 


\section{Chapter 1}

\section{Introduction}

\section{$1.1 \quad$ Problem Statement}

We investigate the practical utility of data structures for biased nearest neighbour search. The nearest neighbour search problem is to preprocess a set of points so that for any query point, the closest point in the set to that query point can be determined quickly. In the biased version of this problem, information on the relative frequency of queries is available, allowing for more common queries to be answered more quickly, improving overall performance.

Nearest neighbour search is of considerable practical interest with applications in geographical information systems, computer graphics, computer vision, signal processing and machine learning, and has received attention from both theoretical and applied computer scientists since at least the early 1970s [30] [9]. A traditional name for this problem is the post office problem, where given a set of sites (the post offices), determine for a given query point which post-office is closest [30].

The biased version of this problem is important because the result distributions for many problems of practical interest are highly skewed. In speech recognition, phonemes, the basic sounds that comprise a language, follow a power law distribution [44] and so some phonemes are much more likely to occur in practice than others. For example, in English, the ten most common phonemes occur roughly 47 percent of the time in spoken language [33]. A geographical information system such as Google 
Maps will receive many more queries for an area around Toronto than it will for an area of the same size around Sioux Lookout.

Although theoretically optimal solutions for nearest neighbour search have existed since the 1990s, these suffer from the so-called "curse of dimensionality" where, as the number of dimensions increases, an increasingly large number of points must be searched until performance in practice is no better than a brute force search of all of the points. Even in low dimensions, nearest neighbour search can be a difficult problem in practice given a large enough point set or the requirement to support a large number of queries in a timely fashion. For these reasons, research has continued to investigate heuristic and approximate approaches to nearest neighbour search.

Recently two groups of researchers $[12,13]$ have proposed a biased data structure, called odds-on trees in [12] that can be applied to a variety of geometrical search problems. It seems as though this data structure may be of practical as well as of theoretical interest.

In a sense, the odds-on tree data structure provides a theoretical basis for the development of heuristics to accelerate geometrical search problems. Since nearest neighbour search is a problem of practical interest with a number of existing heuristic solutions with varying degrees of theoretical justification, it is a natural area in which to apply the odds-on tree with a view to determining its effectiveness in practice.

Our goal is to engineer a practical data structure based on the theoretical results about odds-on trees. This involves design and implementation work as well as careful experimentation to establish correctness and performance characteristics.

Our experimentation investigates the problem of returning a single, exact nearest neighbour. This is a fundamental problem and one to which odds-on trees as described in the literature are immediately applicable. If odds-on trees are not practical in this case, it is not likely they are practical in other cases.

\subsection{Definitions}

Following terminology associated with Voronoi diagrams, we define the set of points for which nearest neighbour searches will be performed as sites. Each site defines an 
area, called a cell, that for all points inside the cell, the associated site is the nearest neighbour for a given metric.

Given a point $p$ its nearest neighbour is the site that is closest to it for a given metric. Its $k$-nearest neighbours are the $k$ sites that are closest to it under a given a metric for a given $k$. A nearest neighbour search is given a query point $p$ determine its nearest neighbour. A $k$-nearest neighbour search is given a query point $p$ return the $k$-nearest neighbours.

A $1+\epsilon$ approximate nearest neighbour search is given a point $p$, return a site that is no more than $1+\epsilon$ times further away from $p$ than the true nearest neighbour.

A $1+\epsilon$ approximate $k$-nearest neighbour search is given a point $p$, return $k$ points such that for each point $r_{i}$ is no further away than $1+\epsilon$ times the distance to the $i$ th true nearest neighbour of $p$.

Entropy is the measure (in bits) of the amount of randomness of a probability distribution. That is, if $p_{i}$ is the probability of the $i$ th member of the set, then the entropy $H$ is defined as

$$
H=\sum p_{i} \log \frac{1}{p_{i}}
$$

A simplex is the intersection of at most $d+1$ halfspaces. A triangle is an example of a simplex in two dimensions, and a tetrahedron is an example of a three dimensional simplex.

A metric is a function $d(p, q)$ defined on the elements of a point set $X$ such that for points $p$ and $q$ of $X$ the following properties hold [38]:

a) $d(p, q)>0$ if $p \neq q$; otherwise $d(p, q)=0$

b) $d(p, q)=d(q, p)$

c) $d(p, q) \leq d(p, r)+d(r, q)$ for any $r \in X$

The Euclidean metric for two points $p$ and $q$ on an $n$ dimensional space is defined as:

$$
\sqrt{\sum_{i}^{n}\left(q_{i}-p_{i}\right)^{2}}
$$

Other metrics are possible, for example, the "Manhattan" or "Taxicab" metric for 
two points $p$ and $q$ is defined as:

$$
\sum_{i}^{n}\left(q_{i}-p_{i}\right)
$$

Unless otherwise stated, all results in this thesis are for the Euclidean metric.

A Gaussian or Normal distribution is defined as:

$$
P(x)=\frac{1}{\sigma \sqrt{2 \pi}} e^{-(x-\mu)^{2} / 2 \sigma^{2}}
$$

The sigma $(\sigma)$ parameter is the standard deviation and the $m u(\mu)$ parameter is the mean of the distribution.

\subsection{Contribution}

In this thesis we present experimental results demonstrating that distribution-sensitive data structures such as the odds-on tree can be used to develop data structures that have performance advantages over standard data structures if provided a suitable probability distribution over the searches.

\subsection{Organization of the Thesis}

The remainder of the thesis is organized as follows: Chapter Two summarizes previous work for biased search, distribution sensitive data structures and nearest neighbour search. Chapter Three describes the design and implementation of the odds-on tree data structure for nearest neighbour search, including a description of alternative designs that were explored. Chapter Four gives details on the experimentation performed and results obtained, and Chapter Five summarizes the results and discusses potential future work. 


\section{Chapter 2}

\section{Previous Work}

In this chapter we examine previous work relevant to odds-on trees. In the first section we examine data structures for nearest-neighbour search. In the second section we present results on biased search and describe the odds-on tree data structure.

Both problems are extremely well studied, so no attempt has been made to be comprehensive and we restrict this survey to commonly used or historically important data structures.

\subsection{Nearest Neighbour Search}

In this section we examine data structures and algorithms for nearest-neighbour search. In particular, we focus on data structures which are based upon orthogonal, axis aligned decompositions of space, as these are the sort of data structure we chose for the odds-on tree implementation.

In general, these data structures work by recursively subdividing an input point set into based upon containment in an axis-aligned hypercube in such a way that at each step either the area of the hypercube or the number of points contained within it or both decrease exponentially at each level.

For example, in a kd-tree, the number of points associated with each child node is half that of the parent node, and in a two dimensional quadtree, the area associated 


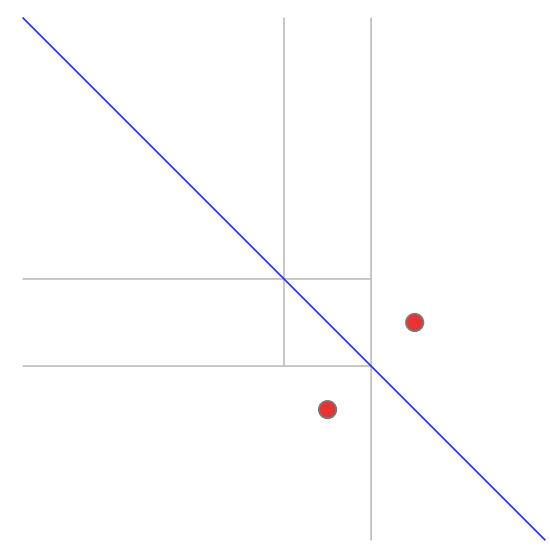

Figure 2.1: Representing Nearest Neighbour Search with Axis Aligned Hypercubes

with each child node is a quarter of that associated with the parent node. The advantage of this approach is that the data structures can be built using only comparisons between point coordinates rather that calculated values and nearest neighbour search can be done using squared distances, both of which avoid problems with numerical and geometrical robustness [41].

The disadvantage of data structures based upon orthogonal decomposition is that it is not possible to do nearest neighbour search in the Euclidean metric with a finite tree, even if there are only two points in the tree, as can be seen in Figure 2.1. The blue line bisecting the two points can not be represented with a finite number of axis aligned boxes.

Because of this limitation, nearest neighbour search in a data structure based upon orthogonal decomposition can not be done solely by point location. The algorithm instead is, given a query point, perform point location for that input point, keeping track of the closest point seen while traversing the tree. Because the nodes in the tree do not represent the area for which the closest point is the true nearest neighbour, it is possibly that the true nearest neighbour is contained in a node which does not contain the query point. To find the true nearest neighbour, it is necessary to backtrack and examine the other sibling nodes for each parent node back to the root of the tree, to see if they in fact contain a point nearest to the query point that the closest point 
found so far.

This backtracking process can be more expensive than the initial search, but can be made much cheaper by doing approximate nearest neighbour search, where nodes are only visited during backtracking if they contain a point which is within an predetermined approximation factor of the distance from the query point to the current approximate nearest neighbour [3]. Even with approximation nearest neighbour search, the cost of backtracking increase as the number of dimensions increases. Empirical evidence suggests that in problems with 16 dimensions or more, using orthogonal decomposition based approaches performs no better on average than a brute force search of all of the sites [2].

Despite these limitations, data structures based upon orthogonal decomposition are widely used and appear to be the fastest solution in practice for small dimensional problems. They are relatively simple to implement and avoid geometric and numerical robustness problems.

Some non-comparison based approaches include locality sensitive hashing [28] where nearby points are hashed to the same cell, or metric trees, where points are sorted based upon distance to a reference point rather than based upon their coordinates. Chapter 4 of the book by Samet [39] features a comprehensive treatment of nearest neighbour search in higher dimensions. The paper by Liu et al. evaluates some of these techniques experimentally [31].

We also restrict ourselves to an in-memory model and do not consider external memory based data structures such as R-trees [25]. Since an odds-on tree is essentially a cache, it is less interesting to consider an external memory representation, although an odds-on tree may be quite useful as an in-memory cache for an external memory data structure.

\subsubsection{General Algorithm for Nearest Neighbour Search}

Nearest neighbour search in data structures based upon orthogonal decomposition is done according to the same general algorithm, based upon the description for kdtrees in [39]. It is described in detail here, rather than being repeated in the sections 
describing individual data structures below.

As described above, a data structure based upon orthogonal decomposition of a $d$ dimensional space is a tree where each node is an axis-aligned box in $R^{d}$. It is constructed based upon a set of input nodes, called sites.

Each child node covers a smaller area than its parent node. The root node covers the area containing all of the input points which were used to construct the tree. An internal node is a node with at least on child, and an external node is a node with no children. Depending upon the data structure, every node may have a site associated with it, or sites may only be associated with external nodes. If a node is associated with a site, it is contained within the node's axis aligned box.

Given a query point $p$, nearest neighbour search is performed by a mixture of depth first search and backtracking. A priority queue, initially containing only the root node is used for backtracking. The priorities are based upon the distance from the $p$ to the nearest edge of the axis-aligned box associated with the node. This backtracking is required due to a finite orthogonal decomposition being unable to exactly represent nearest neighbour search, as described above.

A node is first popped from the priority queue and the distance from the query point to the closest axis is compared to the distance from the query point to the candidate nearest neighbour. If the closest face of the bounding box is further away than the candidate nearest neighbour, then all sites contained within the box associated with the node must also be farther away than the candidate nearest neighbour. Since the priority queue is maintained based upon distance from the query point, it is not necessary to search the remaining nodes and the candidate nearest neighbour is returned as the result to the nearest neighbour search.

If it is necessary to search the node, depth first search is performed on the node to locate the external child node containing the query point $p$. For each child node visited, if there is a site associated with that child, the distance from $p$ to that site is calculated and if it is less than the distance to the candidate nearest neighbour, the site becomes the new candidate nearest neighbour. The distance to the closest face of the bounding box of any sibling nodes is calculated and the are added to the priority queue so they can potentially be visited while backtracking. The depth first search 

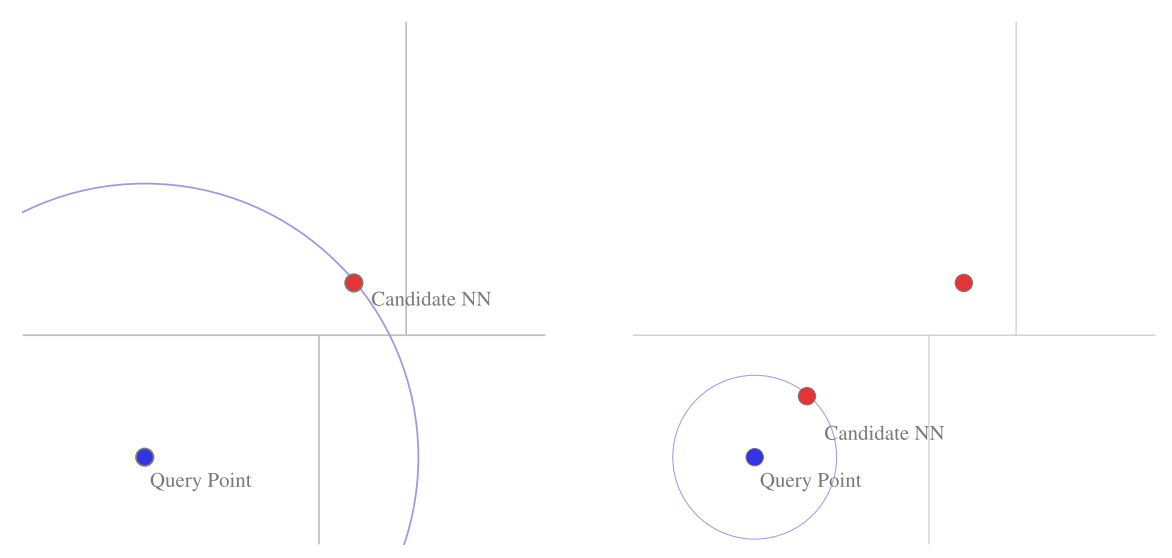

Figure 2.2: Nearest Neighbour Search Algorithm

continues until an external node is visited. At this point, a new node is popped from the priority queue as described above.

These two cases are illustrated in figure 2.2. On the left hand side, the candidate nearest neighbour is further away than the nearest side of the bounding box for a node and so further search is required. On the right hand side, the candidate nearest neighbour is closer than the bounding boxes of the other nodes and so is the true nearest neighbour and the search is finished.

To extend this algorithm to support $k$-nearest neighbour search a priority queue of candidate nearest neighbours can be maintained rather than using a single nearest neighbour. For efficiency, the size of the priority queue can be limited to only hold $k$ nodes. When a node is popped from the priority queue, the distance from th $k$-th nearest neighbour is compared to the distance to nearest face of the bounding box [39].

This algorithm also supports $1+\epsilon$-approximate nearest-neighbour search. In this case, when a node is popped from the priority queue, the distance to the nearest face of the bounding box is multiplied by $1+\epsilon$ prior to comparing it to the candidate nearest neighbour [39]. 


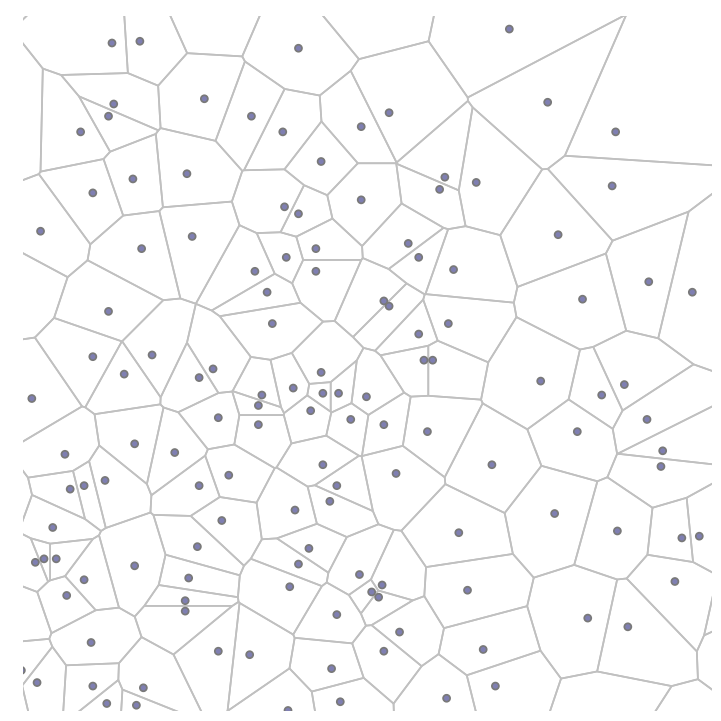

Figure 2.3: Voronoi Diagram

\subsubsection{Voronoi Diagrams}

The Voronoi diagram for a set of sites is the subdivision of the plane (or hyperplane in higher dimensions) into cells, where for every point in a cell, its nearest neighbour is the site associated with the cell. Given a Voronoi diagram, nearest neighbour search is a matter of determining in which subdivision the query point lies.

Efficient algorithms exist for creating Voronoi diagrams in low dimensions:

Theorem. (Voronoi Diagram Construction) The Voronoi diagram for a set of $n$ points in two dimensions can be computed in time $O(n \log n)$. In higher dimensions, the time becomes $O\left(n \log n+n^{\lceil(d+1 / 2)\rceil}\right)$ where $d$ is the dimensionality of the space $[17]$.

\section{Nearest Neighbour Search in a Voronoi Diagram}

Once the Voronoi diagram is constructed, determining the nearest neighbour can be done by performing point location to determine in which Voronoi cell the query point lies. 
In 2D, an efficient data structure for the more general problem of point location in a planar subdivision was described by Kirkpatrick [29]. It based upon first triangulating the subdivision and then removing independent vertices (those which do not share an edge) from the triangulation to create a triangulation with fewer faces. Provided a constant fraction of constant vertices are removed from the triangulation at each step, performing the triangulation recursively will result in a data structure that supports point location in logarithmic time.

Theorem. (Point Location in the Plane) A data structure exists using $O(n)$ storage that allows for planar point location to be performed in $O(\log n)$ time [29].

The same bounds were achieved using different data structures by Sarnak and Tarjan [40] and Edelsbrunner et al. [19].

In higher dimensions, given $n$ hyperplanes, it is possible to solve this problem by constructing a $1 / r$-cutting, that is a collection of simplices that covers the space and is constructed such that each simplex intersects with no more than $n / r$ hyperplanes. Chazelle gives a hierarchical construction procedure for these hyperplanes such that a simplex is eventually fully contained in one of the cells, that can then be labelled and used to perform point location [14].

Theorem. (Point Location in Higher Dimensions) A data structure exists using $O\left(n^{d}\right)$ storage that allows for point location in a d-dimensional arrangement of hyperplanes to be performed in $O(\log n)$ time [14].

Nearest-neighbour search can be performed using Voronoi diagrams and provides efficient algorithms in the plane. In higher dimensions, the time required to build a Voronoi diagram and the space required for the point location data structure both depend upon the dimension of the space in an exponential fashion, which limits both their theoretical and practical interest for this problem.

\subsubsection{Quadtrees}

Quadtrees are based upon recursively dividing n-dimensional space into $2^{n}$ equal sized nodes, with each node in the tree keeping pointers to its children. In the two 


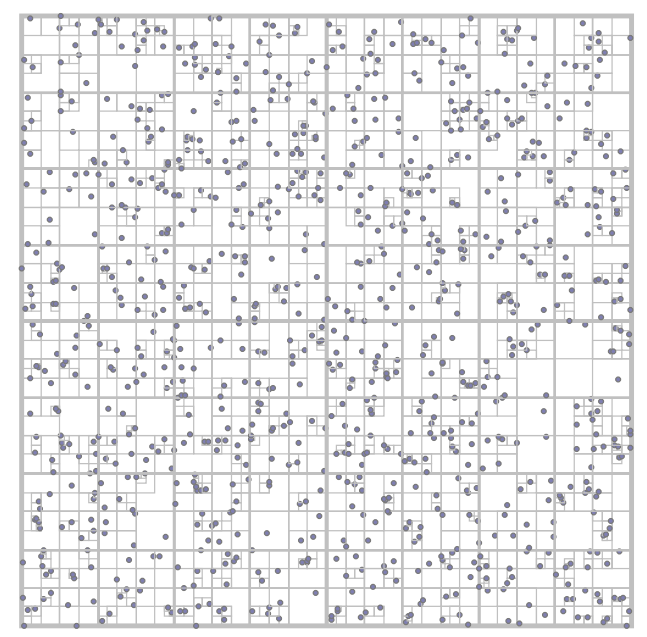

Figure 2.4: Quadtree

dimensional case, this means that space is divided into four quadrants, hence the name quadtrees. Quadtrees were introduced in 1974 by Finkel and Bentley [23].

Quadtrees can be built over a region, in which case the subdivision proceeds until the portion of the region covered by a hypercube has the same value for some property of interest. For example, given an input image, a region based quadtree would continue to subdivide until each square covered an area that had the same colour. Quadtrees over a region are called Trie-based quadtrees in [39] and can be used to approximate Voronoi diagrams.

In this section we use quadtrees that are defined over a set of points, in which case the recursive subdivision is done until each hypercube contains a single point from the point set.

The root of a quadtree is the hypercube containing all of the points, a leaf is a hypercube containing a single point, and the height of a quadtree is the longest path from the root of the quadtree to the leaf of a quadtree [17].

Theorem. (Height of a Quadtree) The height of a quadtree for a set of $n$ points has an upper bound of $\log (s / c)+3 / 2$, where $c$ is the smallest distance between any two 
points in the set, and $s$ is the side length of the initial hypercube containing all of the points. [17].

Note that the height $h$ of the quadtree is not bounded by any function of $n$ and so the running time for algorithms on quadtrees are given in terms of $h$.

Theorem. (Quadtree Construction) A quadtree of height $h$ containing $n$ points can be constructed in $O((h+1) n)$ time [17].

\section{Point Location in a Quadtree}

Point location in a quadtree is performed by recursively visiting nodes starting with the root node. At each step, the child node containing the query point is visited, and the algorithm terminates when a leaf node is reached.

Theorem. (Point Location in a Quadtree) Point location in a quadtree of height $h$ can be performed in time $O(h)$.

\section{Nearest Neighbour Search in a Quadtree}

Nearest neighbour search in a quadtree is performed as described in the section on nearest neighbour search in a bounding volume hierarchy.

Theorem. (Nearest Neighbour Search in a Quadtree) Nearest neighbour search in a quadtree of height $h$ performed in a top down recursive manner needs $\Omega(h)$ time.

\subsubsection{Compressed Quadtrees}

In a compressed quadtree only nodes that contain a point or have at least two child nodes are present in the tree. Compressed quadtrees were first described in [15], and a detailed description can be found in [20].

A compressed quadtree can be created by pruning nodes from an existing quadtree as follows:

1. If a node contains no children and no points, remove it. 


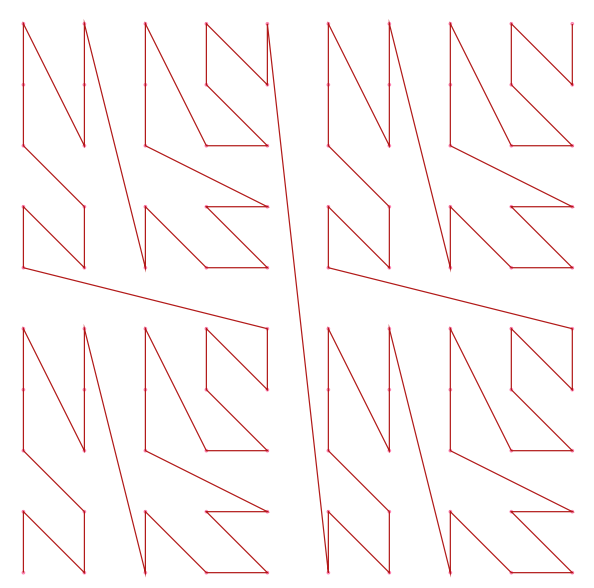

Figure 2.5: Z-Order

2. If a node has a single child node, change the pointer to the node in the node's parent to point to the node's child.

Because this procedure requires first building a regular quadtree, it also requires $\Omega((h+1) n$ time to complete.

This construction procedure can be made more efficient, or at least bounded by $n$, by first sorting the points according to Morton (or z-) order. Morton order is defined by interleaving the bits of each of coordinate of the point, such that the first bit of the first coordinate is followed by the first bit of the second coordinate and so on until one bit has been used from each coordinate. This is then followed by the second bit of the first coordinate [34]. When performed in two dimensions, this results in a z-shaped curve. This interleaving of bits is called the "shuffle" of a point in [11].

The points are sorted according their Morton order. Each two adjacent points in the sorted order form a node in the quadtree that can then be nested by finding the first larger square to the left and the right in the sorted order [11].

Theorem. (Compressed Quadtree Construction) A compressed quadtree containing 


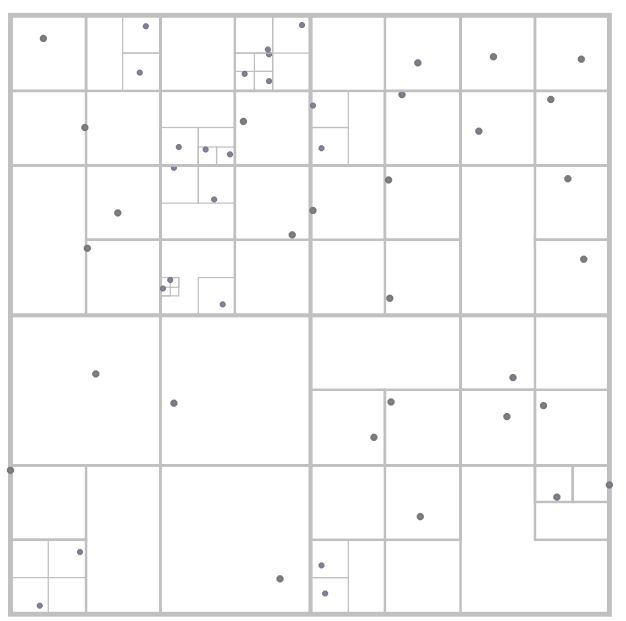

Figure 2.6: Compressed Quadtree

$n$ points can be constructed in $O(n \log n)$ time [11].

Chan [13] showed that the shuffle can be calculated without explicitly interleaving the bits, and Connor and Kumar [16] showed that this can be extended from integer values to IEEE-754 floating point values.

Theorem. (Height of Compressed Quadtree) The height of a compressed quadtree with $n$ points is $O(n)$.

\section{Point Location in a Compressed Quadtree}

Point location in a compressed quadtree is done by following the same algorithm as for standard quadtrees. Since the height of a compressed quadtree is bounded by the number of points, we can bound the time required to perform point location.

Theorem. (Point Location in a Compressed Quadtree) Point location in a compressed quadtree with $n$ points can be performed in time $O(n)$. 


\section{Nearest Neighbour Search in a Compressed Quadtree}

Nearest neighbour search in a compressed quadtree can be done by following the same algorithm as described for bounding volume hierarchies. Again, since the height of a compressed quadtree is bounded by the number of points, we can bound the time required to perform point location by a function of $n$.

Theorem. (Nearest Neighbour Search in a Compressed Quadtree) Nearest neighbour search in a compressed quadtree with $n$ points requires $O(n)$ time.

\subsubsection{Skip Quadtrees}

Skip quadtrees extend the idea of skip lists [37] to two and higher dimensional spaces. In a skip list multiple levels of linked lists exist, with pointers between the levels. The bottom level contains all of the nodes, the top layer is empty, and (in the randomized version of skip lists,) a node present in a given layer is present in the layer above it with specified probability. Corresponding nodes on different levels are then linked. The expected number of layers is logarithmic, allowing for search in $O(\log n)$ time.

Skip quadtrees use essentially the same idea, except each level is a compressed quadtree rather than a linked list, and terminal nodes in the compressed quadtree at one level are linked to corresponding nodes in the lower level tree. We summarize the results are for the randomized version of skip quadtrees. Deterministic results can also be derived using deterministic skip lists [20].

Since each level of a skip quadtree consists of a compressed quadtree with approximately half as many points as that in the level below, we expect to create $O(\log n)$ compressed quadtrees.

Theorem. (Skip Quadtree Construction) A skip quadtree containing n points can be constructed in $O(n \log n)$ expected time [20].

\section{Point Location in a Skip Quadtree}

Point location in a skip quadtree is performed by recursively visiting each level of the quadtree. Since each level is a compressed quadtree, point location is first performed 
in that compressed quadtree. Once the node in the compressed quadtree is located, the link to the corresponding node in the lower level is visited until the bottom level is reached and the node in that compressed quadtree is returned as the result of the point location query. The expected amount of work performed in each level is constant [20].

Theorem. (Point Location in a Skip Quadtree) Point location in a skip quadtree with $n$ points can be performed in time $O(\log n)$ [20].

\section{Nearest Neighbour Search in a Skip Quadtree}

Nearest neighbour search in a skip quadtree is similar to the procedure for searching in a standard quadtree, but the skip structure is used to limit the number of nodes visited.

For a node $p$ removed from the priority queue, the skip structure is used to determine the node $q$ that is the smallest node in the lowest skip level that is equidistant to the query point. It is then necessary to add nodes along the path from $p$ to $q$ into the priority queue for further searching by examining nodes that potentially intersect with the radius from the query point to the candidate nearest neighbour. It is shown in [20] that at most $O\left(\log \frac{1}{\epsilon}\right)$ nodes on the path from $p$ to $q$ need to be added to the priority queue.

Theorem. (Nearest Neighbour Search in a Skip Quadtree) Approximate $1+\epsilon$ nearest neighbour search for d dimensions in a skip quadtree with $n$ points requires $O\left(\epsilon^{1-d}(\log n+\right.$ $\left.\left.\log \epsilon^{-1}\right)\right)$ expected time.

\subsubsection{Kd-Trees}

Kd-trees were developed by Jon Bentley in 1975 as an improvement on quadtrees [9]. Kd-trees are binary search trees for which each non-leaf node divides the point set in half using an axis-aligned line.

Kd-trees are constructed by choosing an axis, determining the median value in the point set for the corresponding coordinate, and dividing the points in two halves 


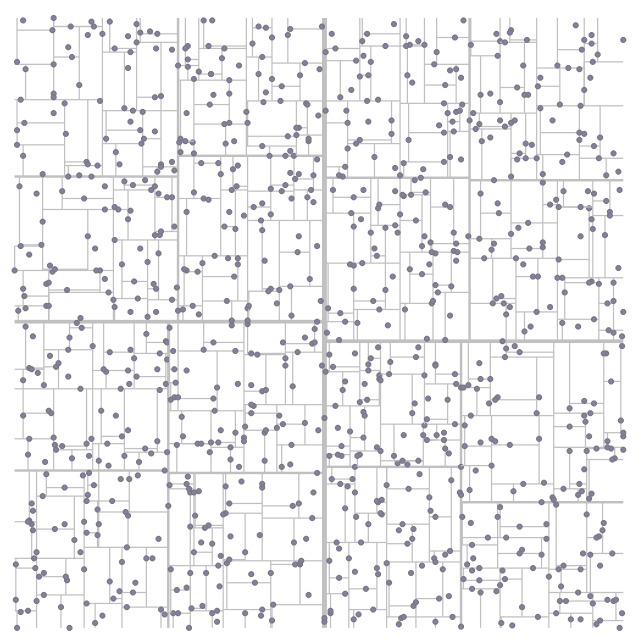

Figure 2.7: Kd-Tree

using the median value. The two child nodes are then built by recursively building kd-trees for each half. In the original paper, Bentley specifies that the axis to split upon is chosen round robin, level by level, but it is possible to develop heuristics that potentially give better splits for specific problems.

Theorem. (Height of a Kd-Tree) The height of a kd-tree with $n$ points is $O(\log n)$.

\section{Point Location in a Kd-Tree}

Point location in a kd-tree can be performed by recursively visiting nodes and comparing the appropriate coordinate of the point to the splitting axis used to split the children for that node, and the visiting the appropriate child node [9].

Theorem. (Point Location in a Kd-Tree) Point location in a kd-tree with $n$ points can be performed in time $O(\log n)$. 


\section{Nearest Neighbour Search in a Kd-tree}

Nearest neighbour search in a compressed quadtree can be done by following the same algorithm as described for bounding volume hierarchies.

Theorem. (Nearest Neighbour Search in a Kd-tree) The expected time for nearestneighbour search in a kd-tree with $n$ points is $O \log (n)$, and the worst case is $O(n)$ [24].

\subsubsection{Balanced Box Decomposition (BBD) Trees}

A balanced box decomposition tree is a data structure invented by Arya et al. [4] that combines advantages of both quadtrees and kd-trees. Like a kd-tree, as one traverses the tree, the number of points associated with a node decreases exponentially, and like a quadtree, the area associated with a node also decreases exponentially. By combining these properties, an optimal data structure for approximate nearest neighbour search is obtained.

Each node in a BBD-tree covers a cell that is the set theoretic difference between an outer box and an optional inner box. Both boxes are axis-aligned. If present, the inner box must either touch the edge of the outer box, or be separated from each edge by at least the width of the box in the direction of the edge, in which case the node is called "sticky". Figure 2.8 reproduced from [4] illustrates "sticky" and "non-sticky" nodes. Each node is an BBD-tree has a bounded aspect ratio that ensures that the ratio of the shortest edge to the longest edge is bounded by some constant.

A BBD-tree is constructed through a sequence of two operations, fair-splits and shrinks. A fair-split is a partition of a box using an axis aligned hyperplane that maintains the aspect ratio bound but does not necessarily result in an even partition of points. A shrink operation divides a node into inner and outer boxes that is used in a manner similar to a compressed quadtree and can ensure an even partition of points [4].

Both fair-splits and shrinks can be performed in time linear in the number of points contained in the cell. A simple strategy for partitioning the points is the midpoint algorithm that performs a fair-split by partitioning the node with a hyperplane 


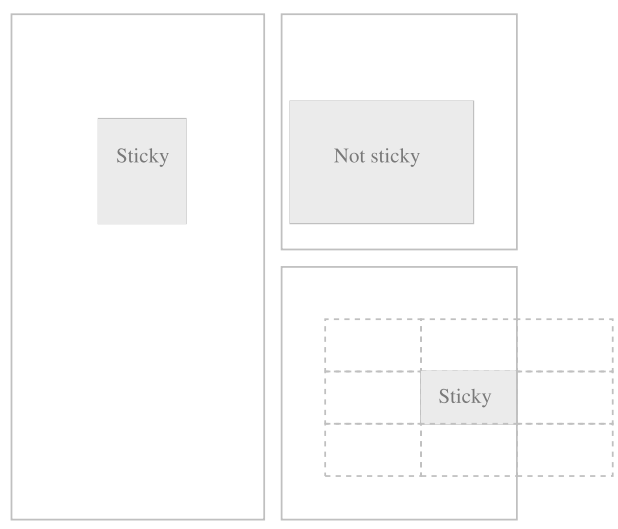

Figure 2.8: Balanced Box Decomposition Tree Nodes

orthogonal to the longest edge of the outer box. A shrink operation is somewhat more complicated, potentially requiring two shrink nodes and one split node to ensure that no child contains more than $2 / 3$ of the points covered by the parent node [4].

To prove properties of the BBD-tree, Arya et al. assume that fair-splits and shrinks are applied alternately as the tree is constructed. In practice, they note that for almost all cases only fair-splits are required [4].

Theorem. (BBD-Tree Construction) A BBD-tree for a d-dimensional space has $O(n)$ nodes and can be build in $O(d n \log n)$ time [4].

\section{Nearest Neighbour Search in a BBD Tree}

Nearest neighbour search in a BBD Tree is performed in a manner similar to search in a kd-tree. A priority queue is maintained of nodes to visit that initially contains the root node. At each step of the search, the closest node to the point is removed from the queue and is recursively searched for the leaf closest to the search point. The distance to the other child nodes encountered in the search is calculated and they are enqueued in the priority queue. The search continues until the distance to the node at the top of the priority queue exceeds the distance to the candidate nearest neighbour. This can be easily extended to find $\mathrm{k}$ nearest neighbours, and to find approximate nearest neighbours rather than an exact ones. 
Theorem. (Nearest Neighbour Search in a BBD Tree) For a fixed constant $c_{d, \epsilon} \leq$ $\left\lceil 1+\frac{6 d}{\epsilon}\right\rceil^{d}$ where $d$ is the dimensionality of the space and $\epsilon$ is the approximation factor, $a(1+\epsilon)$-nearest neighbour search in a BBD Tree can be performed in time $O\left(c_{d, \epsilon} \log n\right)$ time and $k$ approximate nearest neighbours can be found in $O\left(\left(c_{d, \epsilon}+k d\right) \log n\right)$ time.

\subsubsection{Approximate Voronoi Diagrams}

For approximate nearest-neighbour searches, it is possible to improve on the space requirements by creating an approximation to the Voronoi diagram, for instance using quadtrees [26]. The complexity of the calculation is reduced by constructing the Voronoi diagram such that for each cell, any point within it is a $1+\epsilon$-nearest neighbour, rather than an exact nearest neighbour.

Har-Peled [26] provides an algorithm for building an approximate Voronoi diagram that supports nearest-neighbour searches. His construction is complicated, requiring first clustering the input sites, then building a data structure for answering point location in equal ball queries (a technique also used in locality sensitive hashing [28]) and finally using this data structure to build the approximate Voronoi diagram using compressed quadtrees.

Arya et al. [6] extend this to $t, \epsilon$-AVD where each cell in the approximate Voronoi diagram stores $t$ approximate nearest neighbours. Their construction makes use of a well-separated pair decomposition of the sites of the Voronoi diagram. Two sets of points are well separated if each set can be bounded by a ball of radius $r$ such that the distance between the center of each set is at least $2 r$. By decomposing the sites into well-separated subsets, a set of quadtree nodes can be generated that are then stored in a BBD-tree.

We do not describe the construction algorithm in detail as these results are not used in the remainder of the thesis because we only consider exact nearest neighbour search. We note that both $\epsilon$ and $t$ need to be fixed at construction time, which is less flexible than using quadtrees or kd-trees directly.

Theorem. (Nearest Neighbour Search in a Approximate Voronoi Diagram) An approximate Voronoi diagram of size $O\left(\frac{n}{\epsilon^{d}}\right)$ in d dimensions can be constructed that can 
answer $(1+\epsilon)$ approximate nearest neighbour searches in time $O\left(\log \frac{n}{\epsilon}\right)[6]$.

\subsection{Biased Search}

Biased search is a generalization of search where rather than assuming each search result is equally likely, instead a probability distribution over the results is available.

We begin by describing some results from information theory that provide a bound on how well a biased data structure can perform. We then describe some classical results for biased search in a single dimension, and then proceed to some more recent results for higher dimensional search.

\subsubsection{Information Theory}

Lower bounds for search depend upon some results from information theory that are briefly summarized here. Information theory is the study of the fundamental properties of communication systems. A data structure can be seen as a form of communication system because it provides a means of communicating data from the present to the future. A data structure that allows for efficient search is essentially solving the same problem as creating an efficient code with which to transmit a message. The message here is the result of the search.

Based upon earlier work by Ralph Hartley, Claude Shannon [42] defined entropy as the measure (in bits) of the amount of randomness of a set. That is if $p_{i}$ is the probability of the $i$-th member of the set, then the entropy $H$ is defined as

$$
H=\sum p_{i} \log \frac{1}{p_{i}}
$$

Shannon also proved that the efficiency of any communication system is limited by the entropy of the set of messages to be transmitted.

Theorem. (Fundamental Theorem for a Noiseless Channel) Given a source with entropy $H$ (in bits) and a channel with capacity $C$ (in bits per second), then it is possible to encode a source in such a way as to transmit at an average rate of $\frac{C}{H}-\epsilon$ 
symbols per second where $\epsilon$ is arbitrarily small, and it is not possible to transmit at an average rate greater than $\frac{C}{H}[42]$.

To see how a data structure can be viewed as a communication system consider querying a binary search tree for a set of keys. If at each node, a " 0 " or "1" is generated depending upon whether the left or right subtree is taken, then the generated sequence is a code for the key, and we can apply Shannon's theorem to give a bound on the expected number of comparisons required.

For random queries for which the data structure has no information other than the distribution of outcomes, so that $p_{i}$ is the probability of retrieving the $i$-th key, then any data structure for search can not have an expected number of comparisons less than $H$.

Since search time depends upon the expected number of comparisons, the expected search time is bounded by the entropy and $\Omega(H)$ is a lower bound on the search time for any data structure.

\subsubsection{Biased Search Trees}

A biased search tree is a generalization of binary search trees that make use of knowledge of the probability distribution of the search keys to balance the tree.

The initial work on biased search trees focused on the static case, where the query frequencies are known in advance and no deletions and insertions of keys are allowed. This work is summarized in Volume 3 of Donald Knuth's The Art of Computer Programming [30].

Given a set of $n$ keys $K_{i}$ and $2 n+1$ probabilities $p_{1}, p_{2}, \ldots, p_{n}$ and $q_{0}, q_{1}, \ldots, q_{n}$ where $p_{i}$ is the probability that $K_{i}$ is the search item and $q_{i}$ is the probability that the search item lies between $K_{i}$ and $K_{i+1}$, an optimal biased search tree is one that minimizes the expected number of comparisons to search the tree [30].

Each subtree of a optimal biased search tree is itself optimal, and so the problem can be solved through a dynamic programming algorithm, using the following relation: Define $c(i, j)$ as the cost of an optimum subtree with weights $\left(p_{i+1}, \ldots, p_{j} ; q_{i+1}, \ldots, q_{j} ;\right)$ and $w(i, j)$ be the sum of the weights, i.e. $p_{i+1}+\ldots+p_{j}+q_{i+1}+\ldots+q_{j}[30]$. 
Then we have that:

$$
\begin{aligned}
& c(i, i)=0 \\
& c(i, j)=w(i, j)+\min _{1<k<j}(c(i, k-1)+c(k, j)), \text { for } i<j,
\end{aligned}
$$

This recurrence allows us to find all optimal one node trees and then use those to find all optimal two node trees, and so forth, until an optimal search tree has been determined. This minimization is applied to $\frac{1}{6} n^{3}$ values of $k$, but a monotonicity property allows the running time to be reduced to $O\left(n^{2}\right)[30]$.

Theorem. (Construction of an Optimal Static Biased Binary Search Tree) An optimal static biased search tree can be constructed in time $O\left(n^{2}\right)$ and space $O\left(n^{2}\right)$ using dynamic programming [30].

Knuth also proposed two heuristics that run in $O(n \log n)$ time and $O(n)$ space that produce nearly optimal trees:

Rule I: Use the most frequent item as the root of the tree and apply the same rule to the subtrees.

Rule II: Choose as the root the item that results in the weight of the left and right subtrees being as close to equal as possible and apply the same rule to the subtrees.

Mehlhorn [32] analyzed the results of applying these two rules. He provides a simple example where Rule I will produce a tree with expected search time $\Theta(n)$.

His analysis of Rule II shows that the expected search time for item in the tree is bounded from above by the entropy $H$ of the query frequency distribution. If $P_{o p t}$ is the weighted path length of an optimum search tree, and $P_{\text {balanced }}$ the weighted path length of a balanced search tree constructed according to Rule II, then

$$
\log (3)^{-1} \cdot H \leq P_{\text {opt }} \leq P_{\text {balanced }} \leq 2+(1-\log (\sqrt{5}-1))^{-1} \cdot H
$$

Theorem. (Nearly Optimal Static Biased Binary Search Tree) A static biased search tree where the search time for an item is bounded by a constant multiple of the entropy $H$ of the frequency distribution can be constructed in time $O(n \log n)$ and space $O(n)$ [32]. 
Biased search trees allowing insertions and deletions based upon 2-4 and red-black trees were described by Bent, Sleator and Tarjan [8] in 1984. As with 2-4 trees, their data structure relies upon maintaining invariants on the tree during updates through extensive multi-case rules for re-parenting nodes to maintain balance. In this case, the balance condition depends upon the weight of the node, where the weight can be set based upon the probability of its retrieval. If all of the weights are one, the resulting tree is simply a 2-4 tree. Somewhat simpler trees are provided by Feigenbaum and Tarjan [22]. The construction of both data structures requires the consideration of a large number of subcases to maintain balance and so we do not describe them further here.

Theorem. (Construction of a Dynamic Biased Binary Search Tree) A dynamic biased search tree can be constructed in time $O(n)$ that can perform searches in time $O(\log (w))$ where $w$ is the weight of the item searched for and insertions and deletions in amortized time $O(W / w)$ where $W$ is the total weight of all items in the search tree and $w$ is the weight of the item added or removed [22].

\subsubsection{Splay Trees}

An alternative approach to biased search is to use data structures that modify themselves in response to queries rather than insertions or deletions. In this case, rather than specifying the probabilities in advance, the data structure will change itself to match the query distribution.

Splay trees, which were invented by Sleator and Tarjan [43], are a self-modifying binary search tree that perform well in practice. They operate by moving the most recently accessed node to the root of the tree using a process called splaying that rotates items in pairs according to three cases that depend upon the structure of the tree.

Although splaying can be costly for an individual access, in an amortized sense, operations on a splay tree take $O(\log n)$ time.

Theorem. (Splay Trees) Insertion, deletion and search in a splay tree take $O(\log n)$ time in an amortized sense [43]. 
Sleator and Tarjan also showed that splay trees perform within a constant factor of any static optimal search tree over a sequence of accesses, and famously, conjectured that splay trees also perform within a constant factor of any dynamic search tree as well.

Theorem. (Static Optimality of Splay Trees) The total amortized time required for a sequence of $m$ accesses to a $n$ node splay tree is $O(n \log n+m H)$ [43].

It is possible to create biased versions of other, simpler data structures such as randomized search trees (treaps) [1] and skip lists [21] but these do not provide better theoretical performance guarantees than biased search trees and also do not seem to provide better performance in practice than splay trees.

\subsubsection{Planar Point Location}

Looking at biased search in higher dimensions, we first consider the planar point location problem: given a subdivision of the plane and a query point $p$, construct a data structure that allows us to efficiently determine which face of the plane contains $p$. If all of the planar subdivisions and the query point lie on a single line then this problem is the standard one dimensional search problem and the appropriate data structure is a binary search tree.

We discussed the non-biased version of this problem above in the section on nearest-neighbour search in a Voronoi diagram, and noted that a linear space and $O(\log n)$ search time solution existed for this problem.

In the biased case, as with binary search trees, if $p_{i}$ is the probability that the answer to a query is in the $i$ th face and we are given a probability distribution over the faces such that each is chosen with $p_{i}$ then the expected number of comparisons required to locate the query point $p$ is lower bounded by the entropy of the set of faces.

A data structure for biased point location in a triangulation was described by Iacono [27]. It is based on a variation of Kirkpatrick's data structure for solving point location [29], which works by constructed a hierarchical triangulation by selectively 
removing vertices from each level of the hierarchy, until the top level contains a single triangle.

In Iacono's version, the vertices chosen to be removed are such that they are not of large degree and are not incident to a high probability region. Another approach, by Arya et al. matches these space and time bounds [5].

Theorem. (Distribution Sensitive Point Location in a Triangulation) Given a triangulation, it is possible to perform point location in time $O(H+1)$ and space $O(n)$ where $H$ is the entropy of the set of triangles making up the triangulation.

\subsubsection{Biased Range Trees}

Biased range trees are a data structure invented by Dujmovic et al. [18] to solve two-sided planar orthogonal range counting problems. Given a set $S$ and a query point $q_{x}, q_{y}$, the problem is to count all points in $S$ that are above and to the left of the query point $q_{x}, q_{y}$, that is $p_{x} \geq q_{x}$ and $p_{y} \geq q_{y}$. Although the biased range tree solves a fairly restricted problem, it shares many ideas with odds-on trees and is worth considering in detail.

This is a restricted case of the orthogonal range counting problem that allows for a rectangular query area in two or higher dimensions. A data structure invented by Jon Bentley [10] called a Range Tree can solve range search problems in linear space and logarithmic time. A one dimensional range tree is simply a balanced binary search tree, and in $d$ dimensions, one coordinate is chosen to create a binary search tree, and each child node contains a $d-1$ range tree.

Theorem. (Planar Range Search using Range Trees) A range tree for a set of points $S$ of cardinality $n$ in the plane can be built in time $O(n \log n)$ and requires $O(n \log n)$ space. It can report the points in a rectangular query area in time $O\left(\log ^{2} n+k\right)$ time, where $k$ is the number of points in the query area. Using fractional cascading can reduce the query time to $O(\log n+k)[17]$.

Kd-Trees and quadtrees can also be used to solve the orthogonal range counting problem, but with $O(\sqrt{n})$ time and $O(n)$ worst case query time respectively [17]. 
The answer to any range counting query is an integer in $0, \ldots, n$. Let $p_{i}$ be the probability that the answer is $i$. Since we are operating in a comparison based model, Shannon's theorem for a noiseless channel applies and the entropy $H=\sum_{i=0}^{n} p_{i} \log \left(1 / p_{i}\right)$ is a lower-bound on the number of comparisons required. This is not a tight lower bound, and no comparison (or decision) tree is able to meet it in general [18].

A stronger lower bound for the range counting problem can be found by creating an arrangement $A$ of $2 n$ rays originating at each point in the set $S$, with one ray facing to the left and one ray facing down. This partitions the plane into a set of faces $F(A)$.

Now consider a comparison tree to solve the range counting problem. Each leaf of the comparison tree is a rectangle because it is based upon comparisons to coordinates that are axis aligned. These leaf rectangles do not intersect with any of the edges of $A$ because that implies that the answer to the range counting problem would not be consistent for the entire rectangle. This implies that we can use $F(A)$ as a data structure for the range counting problem, and that Shannon's theorem once again provides a lower bound of:

$$
H=\sum_{f \in F(A)} p_{f} \log \left(1 / p_{f}\right)
$$

This lower bound is still not strong enough. To see this consider figure 2.9. The query points are evenly distributed in the $n+1$ red circles. In this case, the query points are always in the same face of $F(A)$, so the entropy above is 0 . A comparison tree based data structure for this problem uses $n+1$ leaves to determine which circle contains the query point, so the lower bound must be greater than $\log (n+1)$.

A Biased Range Tree relies upon two data structures. The primary search tree is based upon a kd-tree. The nodes of the primary tree are associated with regions of space, and the root node covers the plane. Any node in the primary search tree that has depth greater than $\lceil\log n\rceil$ is called a "bad" node. These regions are potentially open on two sides, since the data structure is designed to support 2-sided queries [18].

Each child node in the tree is constructed by removing a horizontal (at even depths) or vertical (at odd depths) strip from region associated with the parent region. 


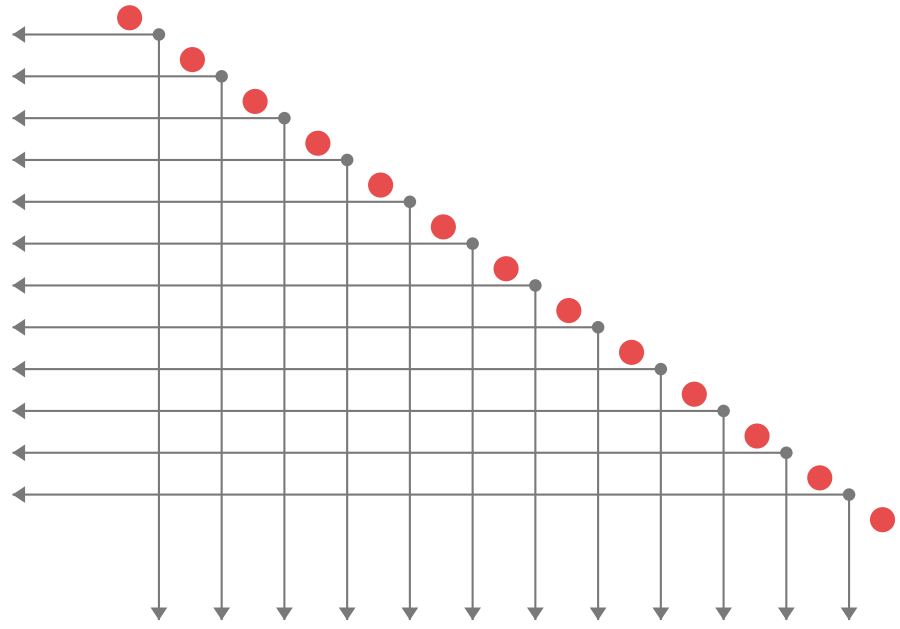

Figure 2.9: The range counting lower bound is not always achievable 
These strips are constructed such that the probability a point being in the region associated with a child node is at most half the probability of the point being in the parent region.

Each node also has two associated data structures, called catalogues, that contain subsets of $S$ sorted by the x- and y- coordinates respectively. Each catalogue is indexed by a biased search tree, and fractional cascading is used to speed up search within the catalogues.

The second data structure is a backup range tree, as described above, that uses $O(n \log n)$ space and can answer range search queries in time $O(\log n)$. This data structure is used to answer queries for which the depth of corresponding node in the primary tree would exceed $O(\log n)$, that is the "bad" nodes.

Search within the biased range tree occurs as follows: the primary tree is first searched to find a node such that the query region defined by $q_{x}$ and $q_{y}$ is contained within the region corresponding to the node. If this node is a "bad" node, then the backup range tree is used to answer the query. Otherwise, the indices are used to locate the node are used to locate $q_{x}$ and $q_{y}$ in the catalogues for the node determine the result of the range counting query, and the tree is walked back to the root, locating the query point in the catalogues of each node along the way, and adding results to the range counting query.

Theorem. (Two-Sided Orthogonal Biased Range Search) Two sided orthogonal biased range search can be performed using space $O(n \log n)$ and expected time $O(H)$ where $H$ is a lower bound on the expected number of comparisons done by any comparison-based data structure, so biased range trees are an optimal data structure for two-sided orthogonal range search [18].

\subsubsection{Odds-on Trees}

Odds-on trees make use of similar ideas of those described above to solve 2-sided orthogonal range searches, but are capable of solving a much larger class of geometric search problems.

Given a probability distribution $D$ over a set of queries, the odds-on tree is capable 
of answering any query drawn from $D$ in expected time $O\left(H^{*}+1\right)$ where $H^{*}$ is a lower bound on the expected cost of any linear decision tree that solves the search problem. In particular, the entropy $H$ of the output $D$ is one such bound for many search problems. A linear decision tree is a search tree where the decision of which child node to follow is based on a linear function.

An Odds-on tree consists of a primary search tree and a backup search tree. The primary search tree is based upon a data structure called partition trees, which are an means of dividing $d$-dimensional spaces into simplices. A simplex is the intersection of at most $d+1$ halfspaces; a triangle is an example of a simplex in two dimensions. The simplices in the partition tree potentially overlap each other.

Theorem. (Partition Trees) Given a set of $S$ points, for any $\epsilon>0$ a data structure called a partition tree can be constructed that uses $O(n)$ storage and can answer counting queries for a search simplex in time $O\left(n^{1 / 2+\epsilon}\right)$ time and report those points in an additional $O(k)$ time, where $k$ is the number of points in the search simplex. A partition tree can be constructed in $O\left(n^{1+\epsilon}\right)$ time [17].

The points used to create this partition are drawn from the query distribution $D$, and the maximum depth of any node in the tree does not exceed a specified level, $\alpha \log n$ for some constant $\alpha$. For each node in the partition tree the area corresponding to the node is searched to see if the answer to the search problem is the same for all points associated with the area. Since the simplices created by a partition tree are possibly overlapping, it is necessary to determine the polytope associated with the node which is not associated with any other node in the tree.

If the result of the search is the same for all points in the polytope, the node is marked as terminal, any children nodes are removed, and the result of the search is stored with the node to answer future searches.

The backup tree can be any data structure capable of answering the original search problem in $O(\log n)$ time.

Search in the tree occurs as follows. Given a query, the tree is searched until either a terminal leaf node is reached, in which case the stored result is used to answer a query, or a non-terminal leaf node is reached, in which case the backup tree is used to 
answer the query [12]. Essentially the same data structure with a different analysis was created by Chan et al. [13].

Theorem. (Odds-on Tree) For any $n$ and $\tau>0$ an odds-on tree with a cache of size $m=n^{\tau}$ of maximum depth $k=\left\lfloor\frac{1}{4} \log _{\frac{r}{3}} m\right\rfloor$, where $r$ is the number of simplices associated with the root node of the odds-on tree, can be constructed in time $O\left(m \log ^{O(1)} n\right)$ plus the cost of $O(m)$ samples from the probability distribution $D$ plus the cost of $O\left(m \log ^{O(1)} n\right)$ searches in the backup tree to determine whether a node is terminal.

The odds-on tree can be answer searches in expected time $O\left(H^{*}+1\right)$ where $H^{*}$ is a lower bound on the expected cost of any linear decision tree that answers the search [12]. 


\section{Chapter 3}

\section{Implementation}

This chapter describes the implementation of an odds-on tree based data structure for nearest-neighbour search.

Partition trees were used in the analysis of odds-on trees. Although they have useful theoretical properties, they are complicated and to our knowledge have never been implemented and so were not considered as the basis of a practical implementation.

Compressed quadtrees and kd-trees lack strong theoretical performance guarantees but they are simple to implement and perform well in practice. In general, kdtrees have better performance guarantees and out-perform quadtrees, but we chose to implement both data structures, as the initial results using kd-trees were not promising and it was possible that quadtrees would have advantages as the basis for an odds-on tree implementation that outweigh their disadvantages in general.

The implementations are in $\mathrm{C}++$. The use of $\mathrm{C}++$ provides great control over memory allocations and the placement of objects in memory, allowing for optimizations. Since it is not garbage collected, experimental results are less variable as the programs will not be interrupted at unpredictable intervals for garbage collection. $\mathrm{C}++$ also has reasonably performant implementations of basic data structures such as vectors and lists in the standard library.

The implementations are parameterized using templates to allow for user defined classes to be used for points, and the underlying data type used for numbers to be chosen. The experiments are done using double precision floating point numbers. 
The heap data structure implementation in the $\mathrm{C}++$ standard library on the development platform was found to have poor performance. Two priority queue implementation were developed based upon the heap data structure, one of which enforced a queue size limitation for use when searching for $k$ nearest neighbours. Both were found to outperform the $\mathrm{C}++$ priority queue implementation based upon the std::vector class and were used in the kd-tree and compressed quadtree implementations.

\subsection{Kd-tree Implementation}

We first describe our implementation of kd-trees. The kd-tree implementation was used as the backup oracle for both the kd-tree and quadtree based odds-on tree, and as the reference implementation of kd-trees for experimentation.

The kd-tree is built as described in chapter two, by choosing a splitting axis round robin, determining the median value in the point set for the corresponding coordinate and dividing the points in two halves using the median value. The two child nodes are then built by recursively building kd-trees for each half.

The most significant optimization made during the implementation is to use a memory pool rather than the system allocator for allocations. This allowed for left and right children of a node to be placed at adjacent locations in memory for better locality of reference and avoided the overhead associated with many small allocations using the system allocator, which resulted in a 50 percent improvement in running time. The implementation was based upon the kd-tree optimization notes in [36].

We performed some experiments comparing our kd-tree implementation to the kd-tree implementation in Mount and Arya's ANN library[35]. Our implementation is slower, but not by a large amount. For instance, running 1000000 queries on a point set of 100000 points, our implementation is eleven percent slower in two dimensions and eighteen percent slower in three dimensions. The ANN library is also as the reference implementation in order to test the correctness of our implementation, by comparing results on sets of randomly chosen points and searches.

In order to support odds-on tree construction, the construction routines in the 
kd-tree were modified to optionally support making a callback function call as each node is constructed. If the callback returns false, the kd-tree construction ends without processing all of the points. This callback mechanism also allows for additional information to be stored at the kd-tree node as required.

\subsection{Kd-Tree Based Odds-on Tree Implementation}

The odds-on tree is built using two point sets. The first point set is used to build the backup kd-tree that is used to answer queries which can not be efficiently answered using the odds-on tree and during construction to answer nearest-neighbour queries while building the cache.

The second point set is drawn from a probability distribution of query frequencies and is used to build the odds-on tree cache. The callback mechanism described above is used to determine if each "corner" of a newly constructed node has the same nearest neighbour. If this is the case, the answer to a nearest neighbour query for any point inside the node would be the same, and it is marked as terminal and the nearest neighbour is stored with the node. This follows from the convexity of Voronoi regions.

A maximum depth for a constructed node is also enforced by the callback function that will end the build process for a node without marking it terminal if the depth of its subtree will exceed a pre-determined maximum.

The build process continues until one of three conditions occurs: all input nodes have been processed, all nodes have been marked terminal, or all remaining nodes exceed the maximum depth specified.

Both the kd-tree and quadtree based odds-on tree implementations handle queries in the same fashion.

To handle queries, the odds-on tree relies upon a locate procedure that searches the cache for a terminal node containing the query point. If a terminal node exists that contains the query point, the stored nearest neighbour is returned. Otherwise, the backup kd-tree is searched for the nearest neighbour. 


\subsection{Quadtree Based Odds-on Tree Implementation}

The Quadtree based odds-on tree is implemented using compressed quadtrees. Two approaches for building compressed quadtrees were investigated. The first, based upon sorting in Morton order did not perform well, and the second, based upon a top down recursive construction was used in the experimentation.

As described in Chapter Two, sorting points in Morton order are sorted in the order in which they would be visited during a depth first traversal of a compressed quadtree. The sorted points were then visited in order to find sequences of points with a common nearest neighbour. Once a sequence was discovered, the end points were used to construct a bounding box, and the "corners" of the bounding box were tested using a backup kd-tree structure. If all of the corners shared a common nearestneighbour, the bounding box is marked as terminal and added to a list of cache nodes. These cache nodes were then used to create a bounding volume hierarchy that is used as the cache for the odds-on tree. This implementation is not competitive with kd-trees and so was abandoned in favour of a standard top down recursive build procedure.

The recursive, top down build procedure for compressed quadtrees was implemented as described in Chapter Two. The root node covers the area containing all of the input points, and each node is split into $2^{d}$ children of equal size. A a compressed quadtree is then built for each child. The build process terminates when only a single point is present in the node or the build callback function indicates that the build should stop. Once the children are built, the child nodes are examined. If only one child node contains children itself, the parent node is replaced by this child, which causes a compressed rather than standard quadtree to be built.

As with the kd-tree implementation, as each node is constructed a callback function is called that allows for the node to be marked as terminal if each corner has the same nearest neighbour. A pre-determined maximum depth is also enforced. Building the compressed quadtree implementation top down is not as fast as sorting by Morton order, but results in a higher quality cache so that cache hit rates are higher and the overall performance is better. 
The query procedure is essentially the same as for the kd-tree based implementation, with a locate procedure attempting to find a terminal node in the cache that contains the query point. If this fails, the backup kd-tree is used to answer the search.

\subsection{Test Harness Implementation}

A test harness for the odds-on tree and kd-tree implementations is used to read test data and output performance metrics.

A $\mathrm{C}++$ program was written to instantiate each of the odds-on tree implementations and the kd-tree implementation. The point, search and sample sets are read from disk to ensure repeatability of results. The results of each search are written to standard output. This allows for comparison of results to a reference implementation. Performance metrics are written to standard error. This allows for search results to be discarded by redirecting standard output to the null device while retaining the performance metrics. Writing results to the terminal causes the searches to perform substantially more slowly.

The test harness outputs the time required to build the tree and the time required to run the queries after the tree is built. The timings are collected using the clock_gettime calls to read the system realtime clock under Linux. The odds-on tree implementations also output the number of "cache hits", that is the number of searches answered by the odds-on tree rather than the backup tree.

The $\mathrm{C}++$ test harness was called by a separate python script which given a directory of sample data, would automatically call the odds-on tree implementation and kd-tree implementation with the appropriate permutations of sample and search sets for each probability distribution over the search sets present. It also provides the capability to randomize the order in which the tests were run, validate that the search results match between the kd-tree and the odds-on tree, and limit the number of runs so that the full set are not performed. These validation and run-limiting features are used to validate the implementations. 


\section{Chapter 4}

\section{Experimental Results}

This chapter describes the experimentation performed to test the odds-on tree implementation, including the experimental design, performance metrics, test platform, and the results of the experimentation.

\subsection{Experiment Design}

The experimental hypothesis is that the odds-on tree provides better nearest neighbour search performance than a kd-tree implementation in cases where the entropy of the point set under a given probability distribution is suitably low.

The following sections describe and justify the experimental parameters chosen for this study.

\subsubsection{Dimension}

The dimension of the point sets is varied across 2, 3, 4, and 8 dimensions. Previous experimental work indicates that dimensions of 16 and above are not suitable for exact search in kd-trees (and by extension quadtrees) and typically require approximate nearest neighbour search for good performance [3]. Pilot experimentation indicated that even at eight dimensions the benefits of using odds-on tree are slight and so higher dimensions were not investigated, as increase in dimension substantially increases 
running times for both the odds-on tree and kd-tree implementations.

\subsubsection{Point Set Size}

The point set is the set of sites that are potential results for a nearest neighbour search. The point sets are generated by sampling a multi-variate uniform distribution on the range $[-1,1)$ for each dimension.

The number of points in the point set is varied across 1000, 5000, 10000, and 50000 points. Larger point set sizes were tried during pilot experimentation but substantially increased run times without giving significantly different results.

\subsubsection{Search Set Entropy}

The search set is the set of query points that are used to test the odds-on tree and kd-tree implementations.

A multi-variate Gaussian distribution is used to generate the search set. The mean for each dimension is 0 , so the search set is distributed around the origin. The standard deviations (or sigma) used are 0.5, 0.25, 0.1, 0.05, and 0.01 .

The figures below show an example of the point and sample set distributions for each sigma value. The sample set is a smaller set drawn from the same distribution as the search set and so illustrates the search set distribution.

For each case the search set consists of 100000 points. In pilot experiments this size ensured long enough running times to make meaningful comparisons between experimental cases without requiring an excessive amount of time to perform all of the experiments. If too few searches are used the run times may end up being within fractions of a second of each other and so much more likely to be affected by noise.

The experimentation in this thesis is done using synthetic datasets due to the difficulty in finding existing datasets that include suitable probability distributions over the searches . This also makes it difficult to demonstrate that the search distributions chosen reflect "real-world" problems.

Although it is possible to take existing datasets and attempt to derive or create typical search distributions for them, this would essentially still be using synthetic 


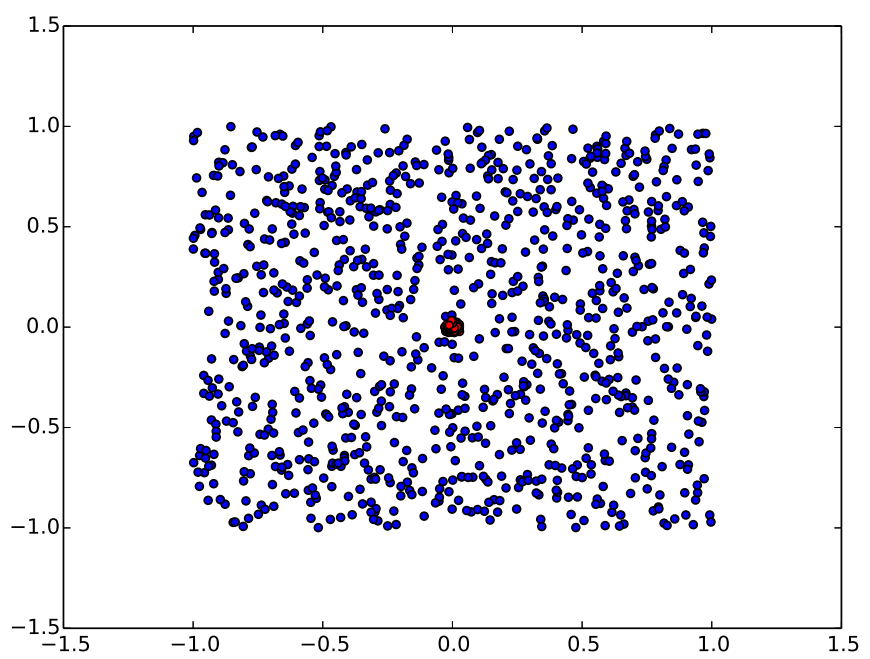

Figure 4.1: Points and sample distributions $(2 \mathrm{~d}$, sigma $=0.01)$

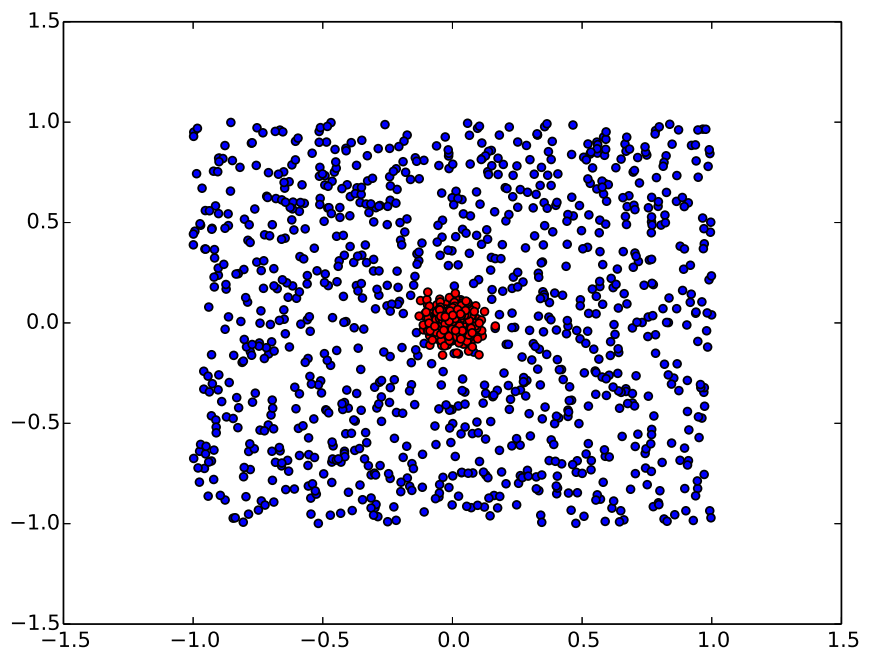

Figure 4.2: Points and sample distributions $(2 \mathrm{~d}$, sigma $=0.05)$ 


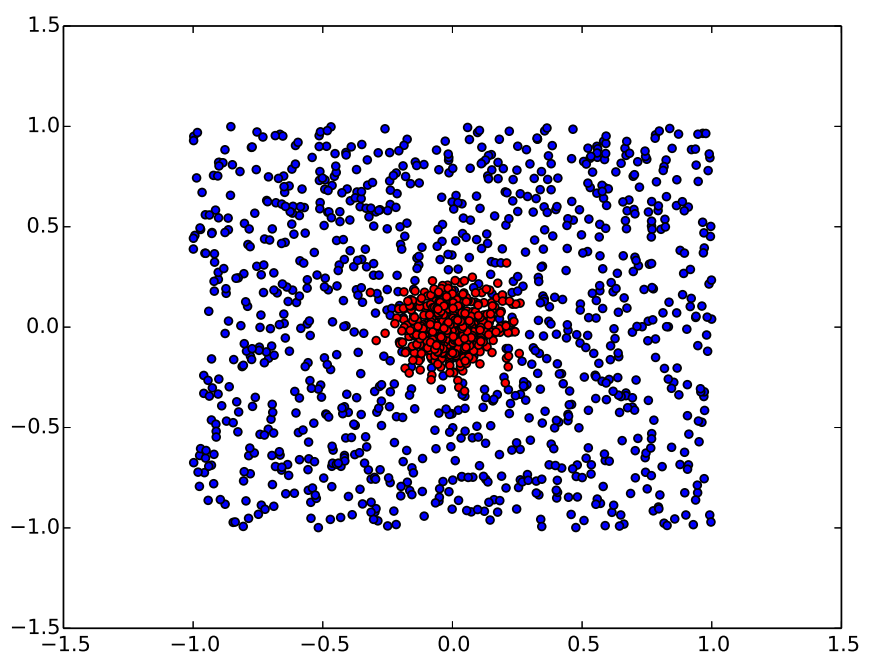

Figure 4.3: Points and sample distributions $(2 \mathrm{~d}$, sigma $=0.10)$

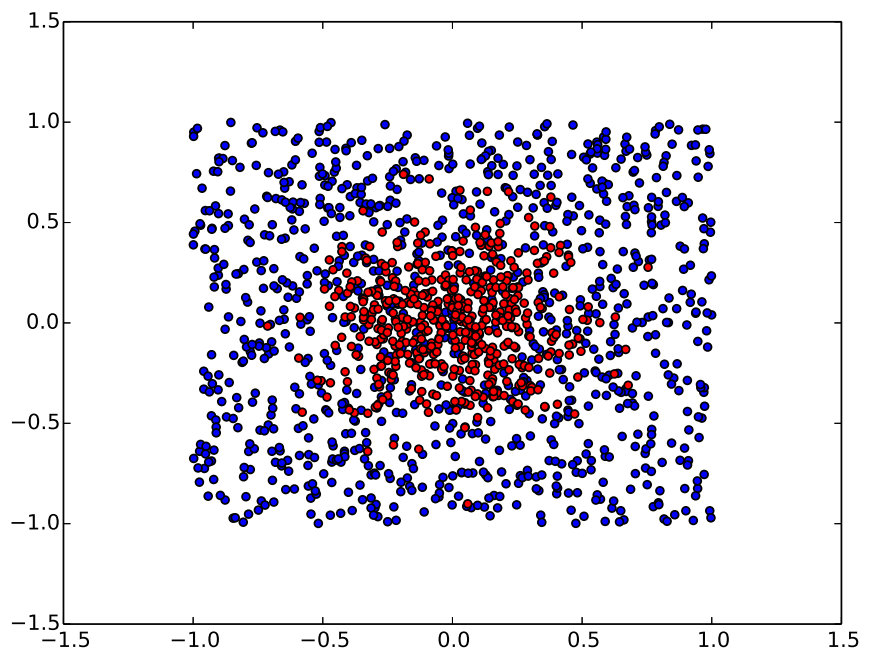

Figure 4.4: Points and sample distributions $(2 \mathrm{~d}$, sigma $=0.25)$ 


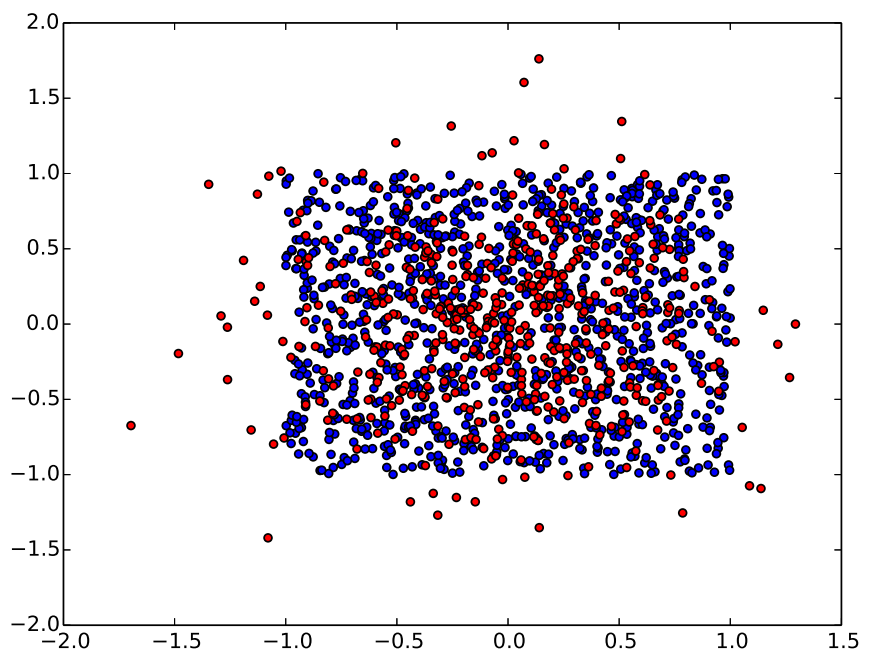

Figure 4.5: Points and sample distributions $(2 \mathrm{~d}$, sigma $=0.50)$

data because the primary determinant of odds-on tree performance is the entropy of the sites given a probability distribution over the searches.

\subsubsection{Sample Set Size}

The sample set is a set of searches used to build the odds-on tree. Each sample set is created by sampling the same probability distribution used to create the search set. A different sample set is generated for each run. Example sample sets are shown in the figures above.

The sample set set size used is $0.5 n, n$, and $2 n$, where $n$ is the cardinality of the point set. Pilot experimentation showed that these values provide a reasonable indication of the performance impact of sample set size.

\subsubsection{Maximum Build Depth}

The construction of the odds-on tree is controlled by a parameter that determines how "deep" the recursive build will proceed before terminating. This parameter will 
be set be varied between $\log (n), 1.5 \log (n)$, and $2 \log (n)$ where $n$ is the cardinality of the sample set. Values less than $\log (n)$ and greater than $2 \log (n)$ did not have a substantial impact on results in the pilot experimentation.

\subsubsection{Number of Runs}

Each experimental configuration is repeated ten times with a different sample set in each case. During pilot experimentation, this was shown to generate statistically significant differences between configurations for nearly all cases examined (t-test, $p<0.0001)$.

\subsection{Measuring Performance}

\subsubsection{Benchmark Platform}

The experimentation is done using a virtualized host provided by a third party. This allows for easy duplication of hardware to run experiments in parallel and aids in repeatability. Although instance types are eventually retired by providers, physical hardware is likewise only available for limited period of time.

The benchmark platform is a Digital Ocean droplet with a $2.4 \mathrm{GHz}$ processor, 1 GB of RAM, and 30 GB SSD secondary storage. During pilot experimentation, the digital ocean instance was found to be considerably faster and so more cost-effective than an Amazon Web Services EC2 Medium Compute Optimized instance, with two 64-bit processors and 1.7GB of RAM. This is likely due to the Amazon instance type not providing SSD backed storage and so becoming I/O bound reading experiment data.

\subsubsection{Performance Metric}

The primary performance metric is running time. As mentioned above, the time required to build the tree and the time required to run the queries are measured separately, allowing these times to be compared independently of the overall running 
time. This is done because the effect of build time can be made arbitrarily small by increasing the number of queries performed.

A secondary performance metric for the odds-on tree is the cache "hit rate". This is the number of queries answered by the odds-on tree as opposed to the backup tree. This allows for comparison between the kd-tree based and quadtree based odds-on tree implementations.

\subsection{Results}

This section contains the results and analysis for each experimental parameter described above and concludes with a comparison of the quadtree and kd-tree based odds-on tree implementations and their performance relative to the kd-tree.

\subsubsection{Dimension}

An increase in dimension caused a corresponding increase in the query time of the odds-on tree and kd-tree implementations. It also caused the relative performance of the odds-on tree to become worse. In two or three dimensions and small search set sigma, the odds-on tree outperforms the kd-tree. In eight dimensions, even with a favourable search set sigma, the kd-tree equals or outperforms the odds-on tree in query time. Typical results for a quadtree based odds-on tree are shown in figure 4.6. This decrease in performance is likely caused by the decreased effectiveness of a fixed sample size as dimensionality increased.

Build times likewise became worse for the odds-on tree implementations as dimensionality increased. The impact on the kd-tree build time was negligible.

\subsubsection{Point Set Size}

To see the impact of point set size, the build depth and sample sigma are held constant and the build and construction times are plotted for each point set size.

For the kd-tree based implementation increasing the point set size increases build time and query time and query time variance. The kd-tree query time increases, but 


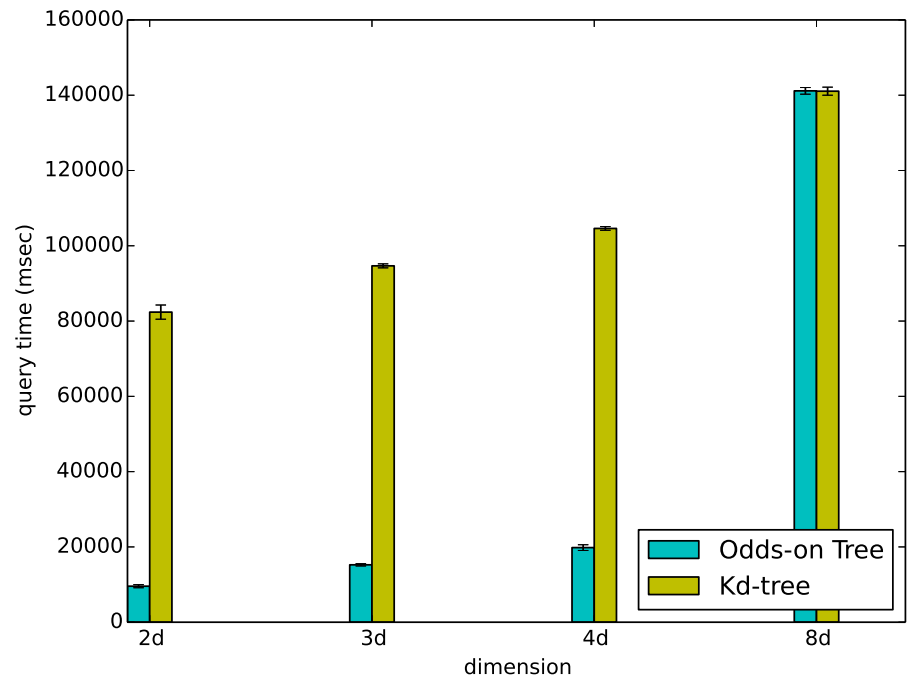

Figure 4.6: Query Time $($ pts $=50000$, sample $=25000$, sigma $=0.01)$

the build time is not significantly impacted at the point set sizes tested, in comparison to the odds-on tree implementation.

For the most part the relative query performance of the kd-tree based odds-on tree and kd-tree did not change with increased point set size. If the odds-on tree outperformed the kd-tree for small point set sizes, it also did so for large point set sizes. There were a few exceptions at larger search set entropies and higher dimensions.

The results for the quadtree based odds-on tree follow the same pattern, with increased point set size increasing the overall running time, but not changing the relative performance of the odds-on tree and the kd-tree. Typical build time results are shown in figure 4.9 and construction time results in figure 4.10. The construction time required for the kd-tree is negligible compared to the time required for the oddson tree. 


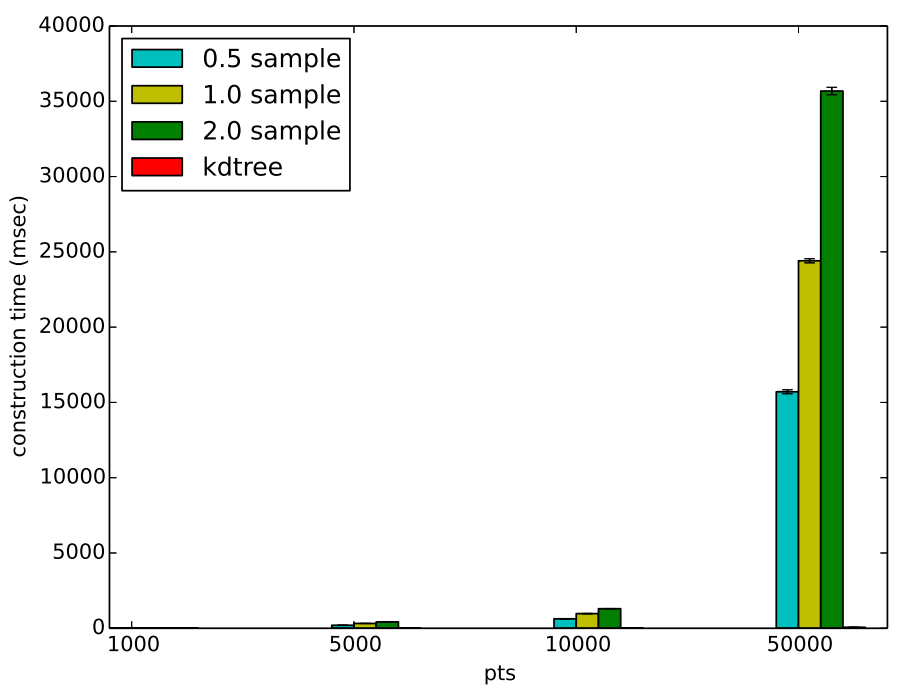

Figure 4.7: Construction Time (kd-tree, 2d, sigma $=0.01$ )

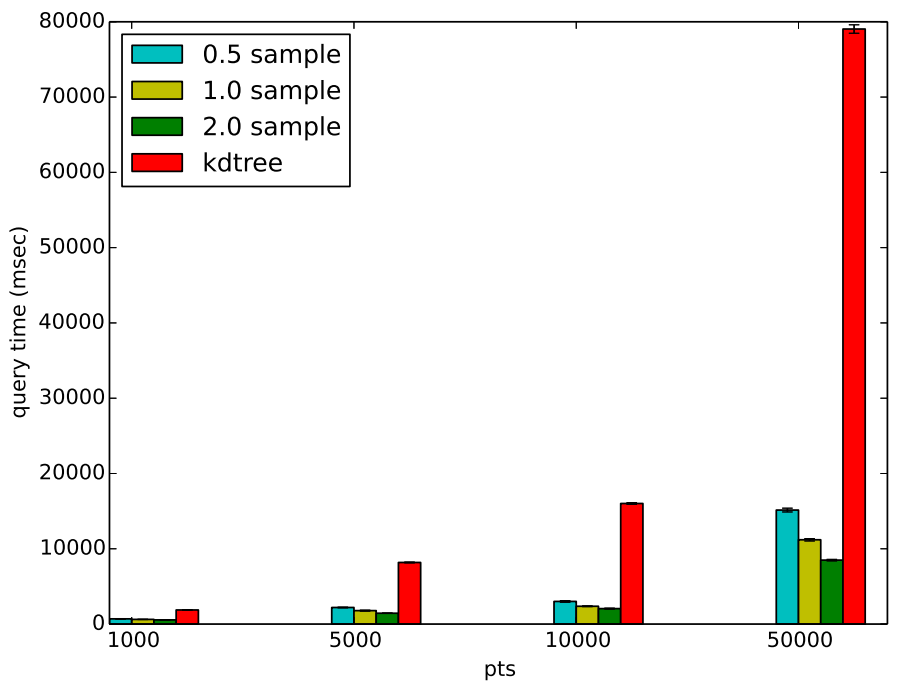

Figure 4.8: Query Time (kd-tree, 2d, sigma $=0.01)$ 


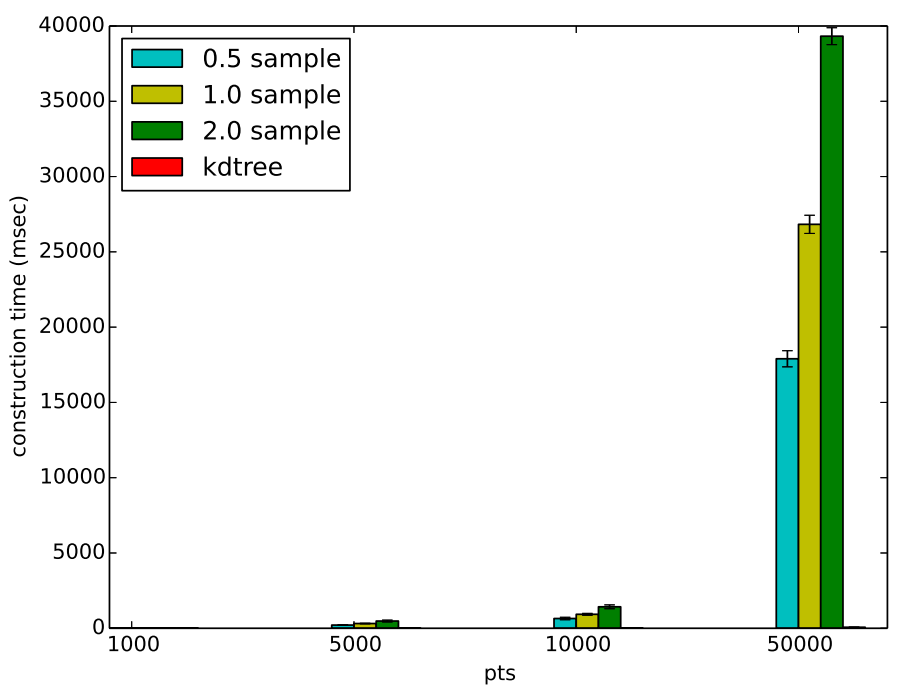

Figure 4.9: Construction Time (quadtree, 2d, sigma $=0.01$ )

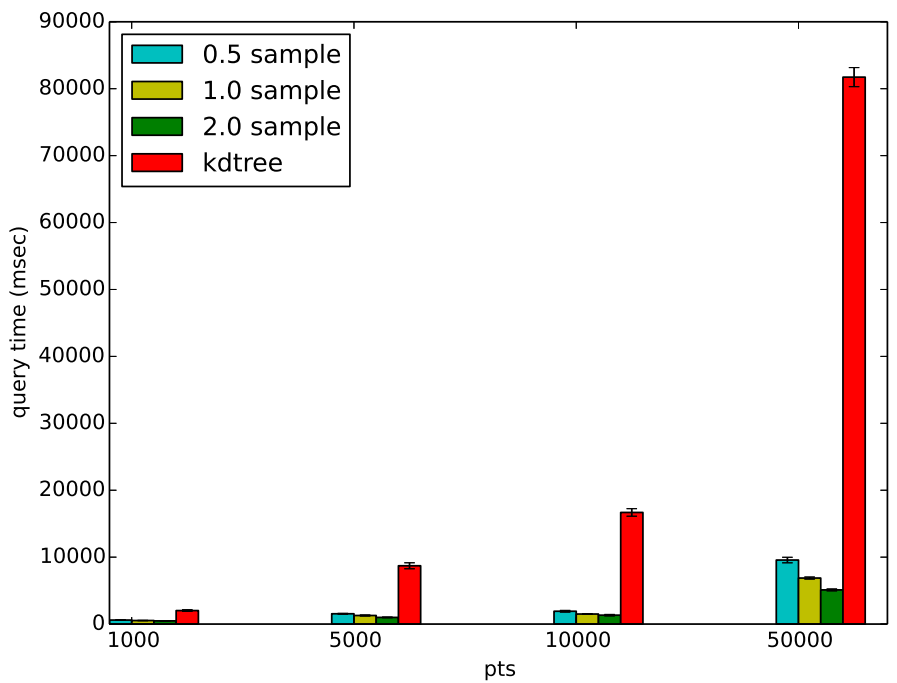

Figure 4.10: Query Time (quadtree, 2d, sigma $=0.01$ ) 


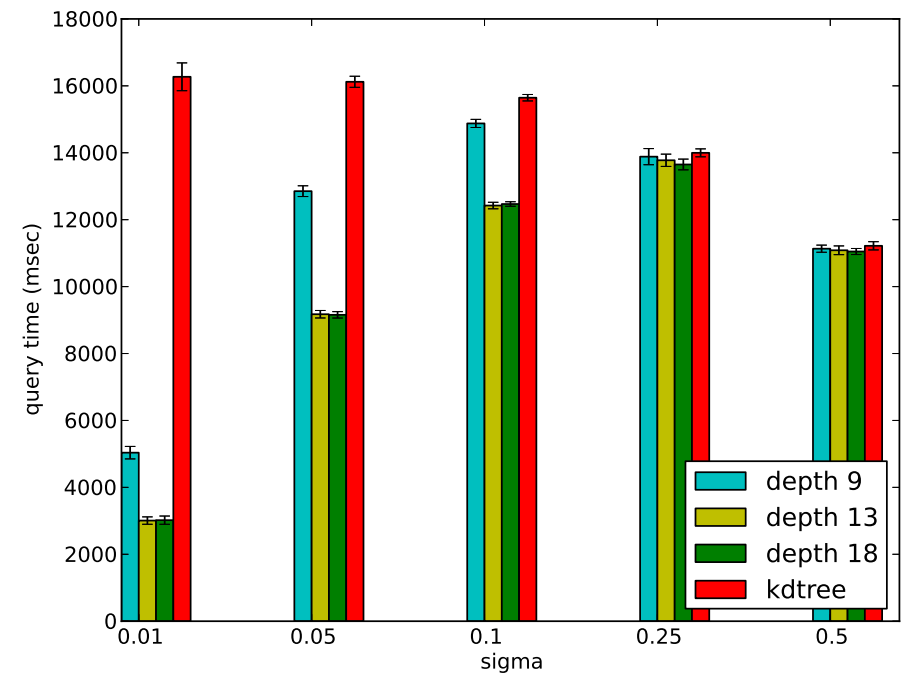

Figure 4.11: Query Time (kd-tree, 2d, pts=10000, sample=5000)

\subsubsection{Search Set Entropy}

Increasing sigma value causes the performance of the odds-on tree to deteriorate. At small sigma values, the odds-on tree performs significantly better than the kd-tree. As sigma increases, the odds-on tree begins to perform worse until its performance is more or less equivalent, indicating that almost all queries are being handled by the backup kd-tree rather than the odds-on tree itself.

As sigma increases, the performance of the kd-tree itself also improves. This is due to the kd-tree doing less backtracking to determine if the candidate nearest-neighbour is in fact the actual nearest-neighbour at larger sigma values. For sigma $=0.25$, the kd-tree does 17 percent less backtracking, and for sigma $=0.5$ the kd-tree does 35 percent less backtracking. This is in turn a side effect of the searches being centered around the origin. As sigma increases, the searches are more likely to fall on the periphery of the kd-tree, meaning fewer nodes inside the kd-tree need to be visited while backtracking to find the nearest neighbour. Figure 4.11 shows query time results for a $2 \mathrm{~d} \mathrm{kd}$-tree based odds-on tree.. 


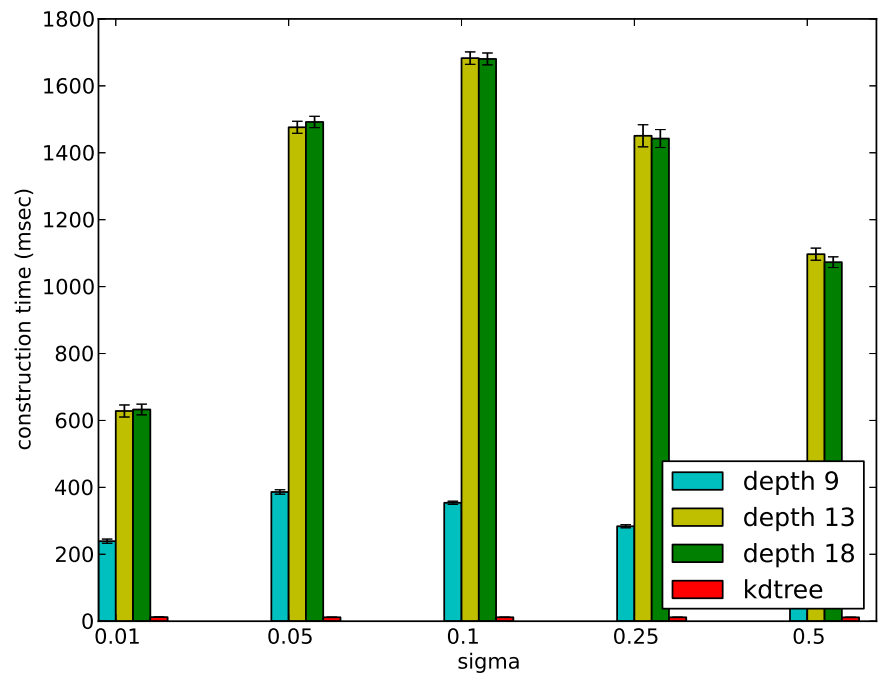

Figure 4.12: Construction Time (kd-tree, 2d, pts=10000, sample=5000)

The construction time for the kd-tree based odds-on tree implementation worsens as sigma increases until it reaches a maximum at a sigma of 0.1. At this point, the construction time begins to decrease. This decrease matches the decrease in query time for the kd-tree at those sigma values. Since constructing the odds-on tree requires a large number of kd-tree based nearest neighbour searches, the improved kd-tree performance also benefits the construction time for the odds-on tree. Figure 4.12 shows construction time results for a $2 \mathrm{~d}$ kd-tree based odds-on tree..

The observed decrease in performance with increasing sigma values is due to a larger odds-on tree being built. The number of terminal nodes remains relatively small while the overall size of the odds-on tree and the number of nearest neighbour queries required increases substantially. Typical results are shown in figure 4.13.

\subsubsection{Sample Set Size}

The sample set size has a direct impact on build and query times. Increasing the sample set size requires more time to build due to additional nearest neighbour queries 


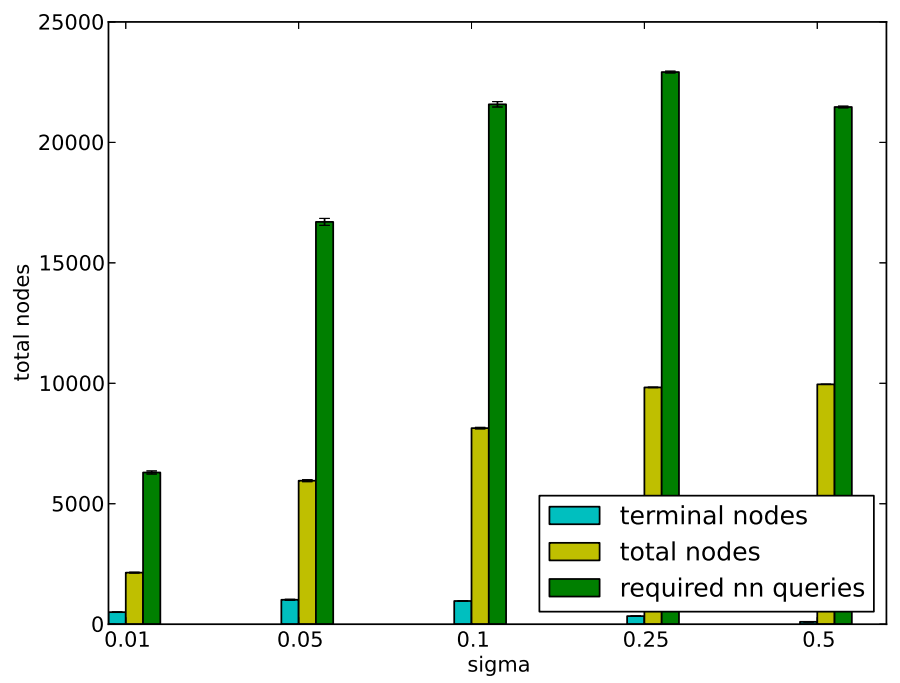

Figure 4.13: Node Counts (kd-tree, 2d, pts=10000, sample $=10000$, build depth=13)

during the cache construction, but usually results in a better cache, causing a corresponding decrease in query time. An example of typical build time results are shown in figure 4.15 and query time results are in figure 4.14 .

Because changing sample set size is a trade off between build time and query time, it makes sense to present results for total time. A typical result for overall running time is shown in figure 4.16. For lower sigma values the higher quality cache that results from a larger sample set size results in a lower overall running time. As sigma increases, this benefit decreases until performance becomes roughly equivalent for all sample set sizes, and before finally becoming worse for larger sample set size, at which point the kd-tree outperforms the odds-on tree.

Increasing the sample set size increases both the number of terminal nodes and the total number of nodes in the odds-on tree as can be seen in figure 4.17. The overall ratio of terminal nodes to total nodes is highly dependent on the search set entropy. At low entropy, increasing the sample set size increases the number of terminal nodes while maintaining a small overall odds-on tree size. This means that the odds-on tree is quick to search, and searching it is likely to result in finding a terminal node. This 


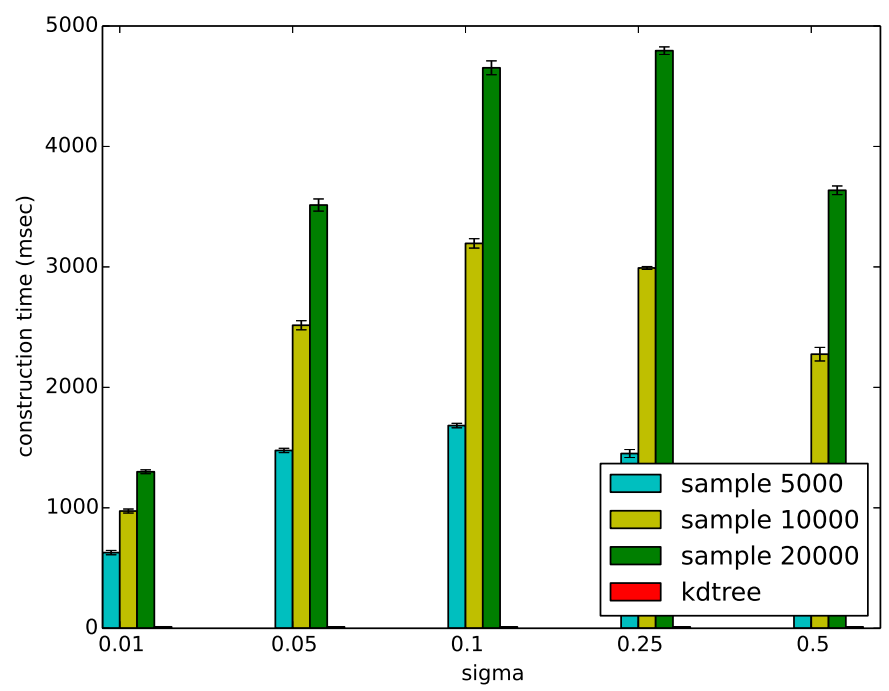

Figure 4.14: Construction Time(kd-tree, 2d, pts=10000)

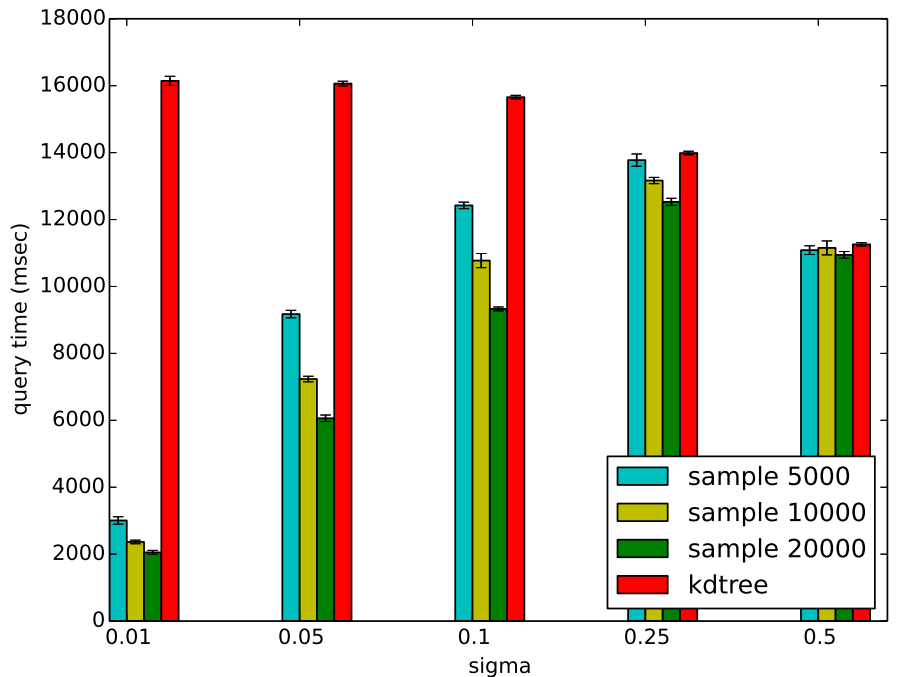

Figure 4.15: Query Time(kd-tree, 2d, pts=10000) 


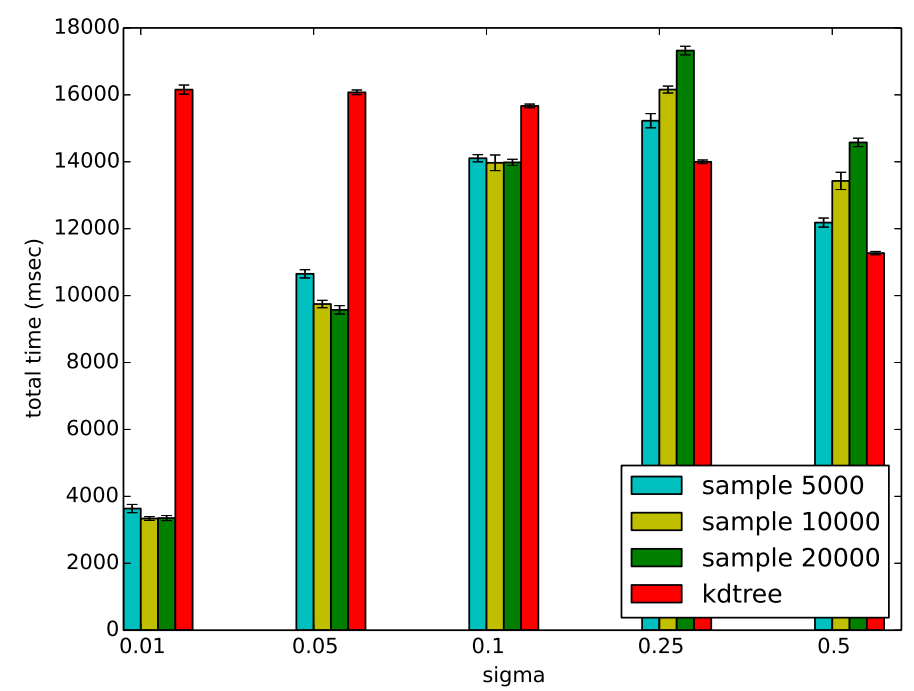

Figure 4.16: Total Time(kd-tree, 2d, pts=10000)

is not the case at higher search set entropy values.

\subsubsection{Maximum Build Depth}

The maximum build depth parameter limits how deep the odds-on tree will be built, which affects both construction time and query time. The smallest build depth value $(\log (n)$, where $n$ is the point set size) investigated typically results in faster construction time but slower query times and slower overall performance.

The results for the other two build depth values $(1.5 \log (n), 2 \log (n))$ are typically very close together. Example results for build time are shown in figure 4.18 and for query time are shown in figure 4.19 .

As with sample set size, build depth is a trade off between build time and query time. A typical result for overall running time is shown in figure 4.20. In this case the trees with greater depth are faster for the sigma $=0.01$ condition, but the additional build time ends up making them slower overall for greater sigma values.

In comparison to the sample set size, the maximum build depth parameter has 


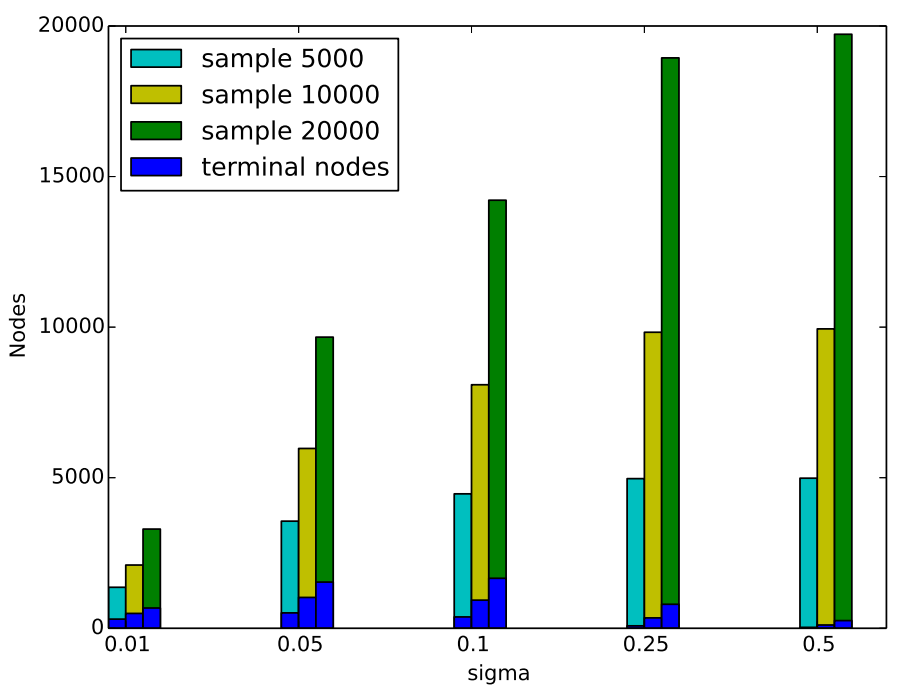

Figure 4.17: Node Counts (kd-tree, 2d, pts=10000)

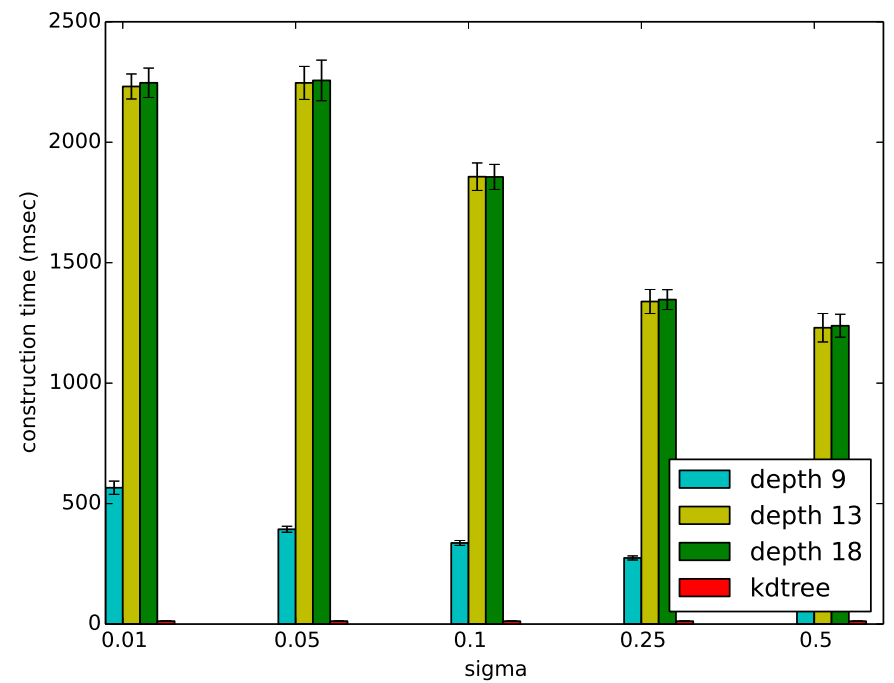

Figure 4.18: Construction Time(kd-tree, 3d, pts=10000, sample $=5000)$ 


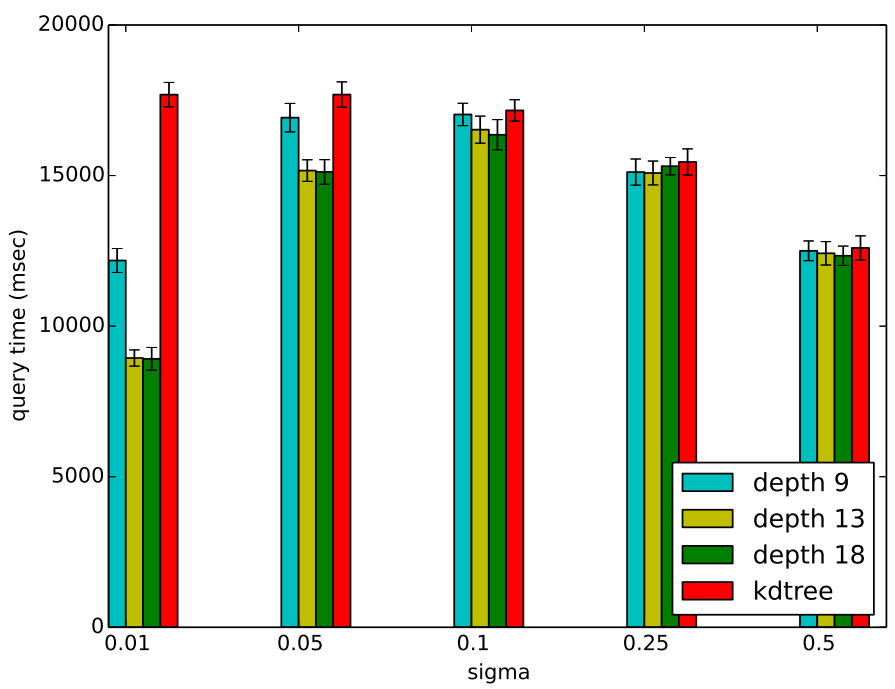

Figure 4.19: Query Time(kd-tree, 3d, pts=10000, sample=5000)

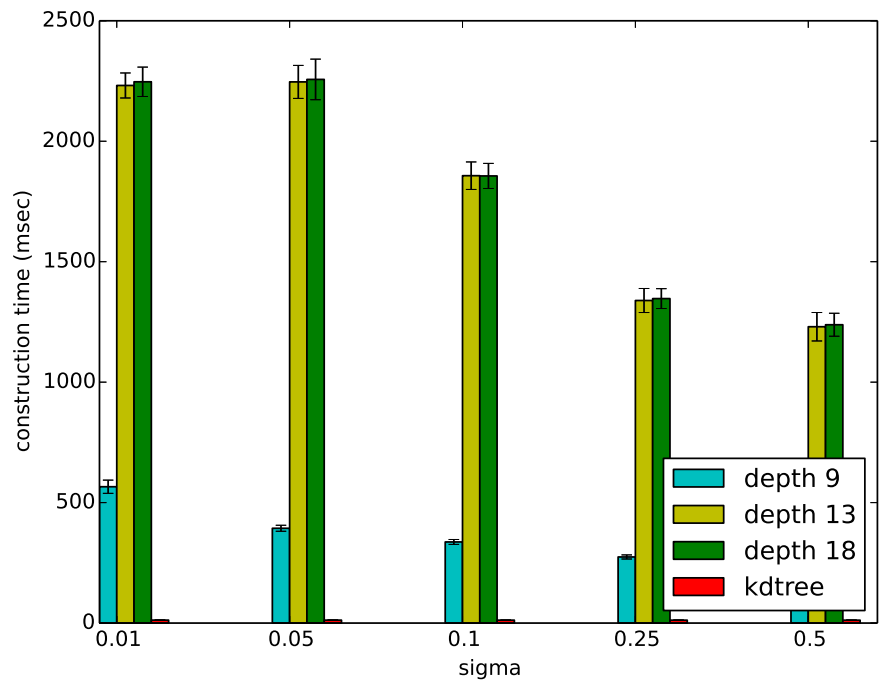

Figure 4.20: Total Time(kd-tree, 3d, pts=10000, sample=5000) 


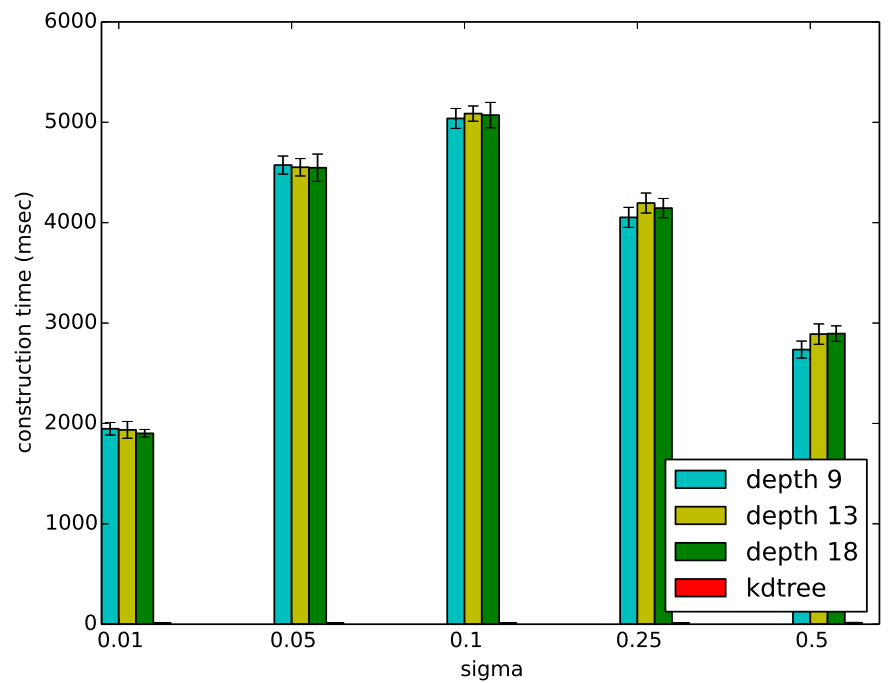

Figure 4.21: Construction Time(quadtree, 3d, pts=10000, sample=5000)

a much less predictable effect on the performance of the odds-on tree. Based upon the results here, it seems best to set it at a moderate value, say between $1.25 \log (n)$ and $1.5 \log (n)$ and use sample set size to make trade offs between build time and construction time of the odds-on tree.

For the quadtree based odds-on tree there is a lot of variance in the running time and the build depth parameter does not seem to have a significant impact on either build time or construction time. As with the kd-tree based implementation, it seems best to set it at moderate values and use the sample set size, which has more predictable impact on performance.

\subsubsection{Quadtree vs. Kd-Tree Based Implementation}

Pilot experimentation did not show either the kd-tree or quadtree based implementation to be consistently better than the other. In worst case analysis, kd-trees are expected to exhibit better performance than compressed quadtrees for nearest neighbour search, but this is not necessarily the case for the experimental conditions 


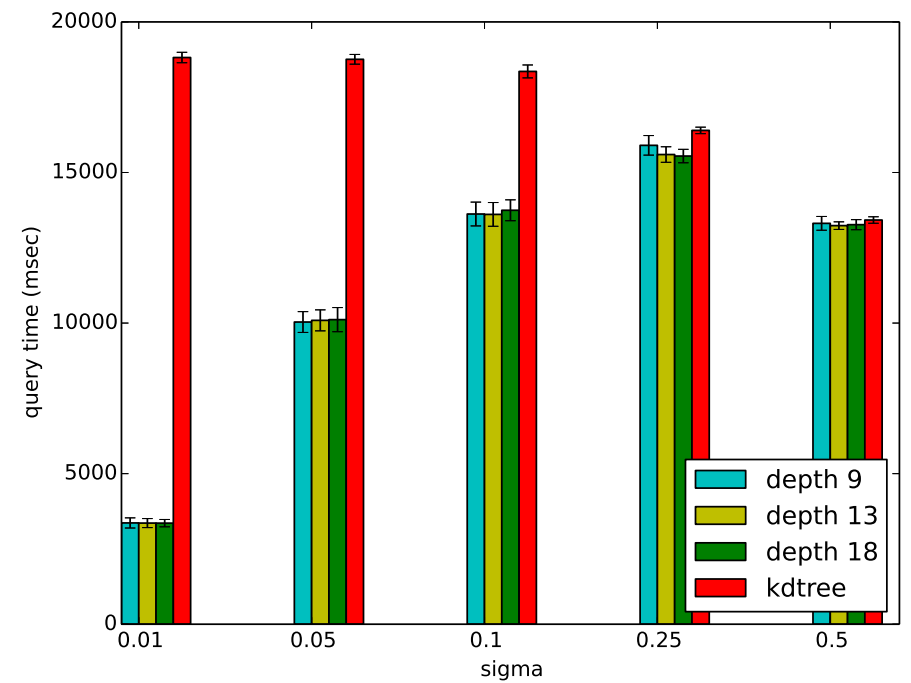

Figure 4.22: Query Time(quadtree, 3d, pts=10000, sample=5000)

investigated in this thesis.

The quadtree build times are generally worse than the kd-tree based build times. A kd-tree can be built efficiently by sorting in place, but constructing a compressed quadtree using a recursive top-down procedure requires allocating space for new nodes, copying points into them, and then potentially pruning them after the face. It is possible to build compressed quadtrees based upon sorting points (see the section on z-order above) but pilot experiments found that constructing quadtrees this way is faster, but resulted in lower quality caches so that overall running time was slower.

For query time it is found that for conditions under which the odds-on tree performs well (sigma $\leq 0.1$ ), the quadtree based implementation will outperform the kd-tree based implementation.

Comparing total run times, the quadtree based implementation outperforms the kd-tree based implementation for low entropy conditions (sigma $\leq 0.05$ ) figure 4.25 . This is not the case in higher dimensions. The quadtree based implementation is substantially worse than the kd-tree base implementation for the $d=8$ case (see figure 4.26). Quadtrees exhibit best performance in small dimensions. This is in 


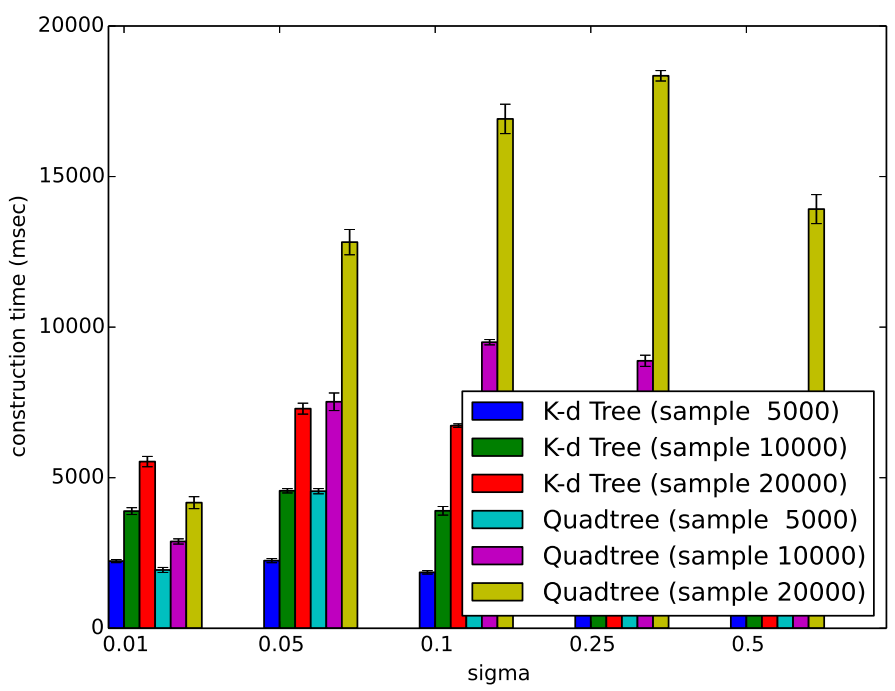

Figure 4.23: Build Time(3d, pts=10000)

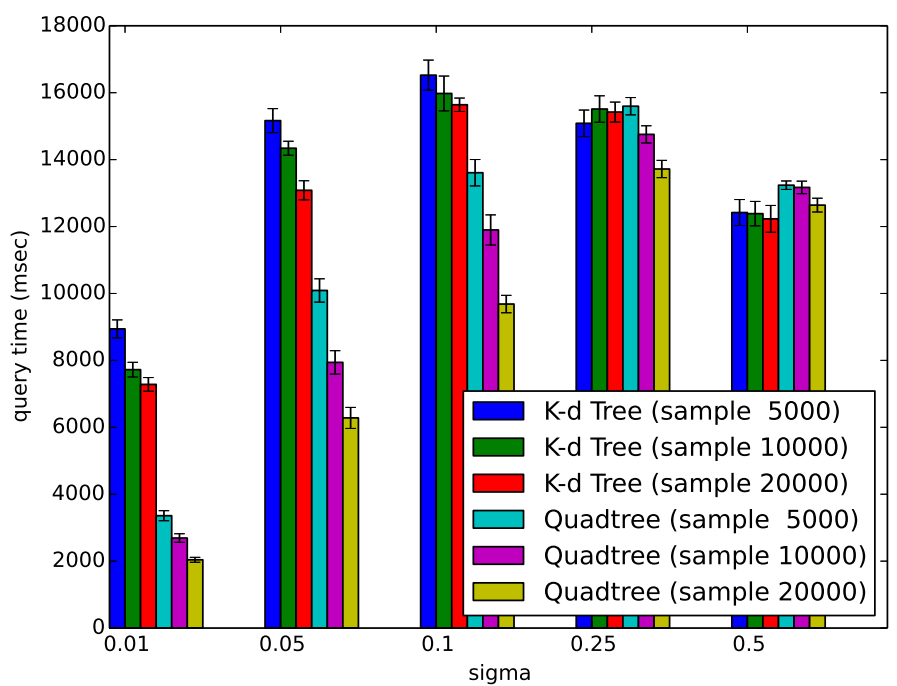

Figure 4.24: Query Time(3d, pts=10000) 


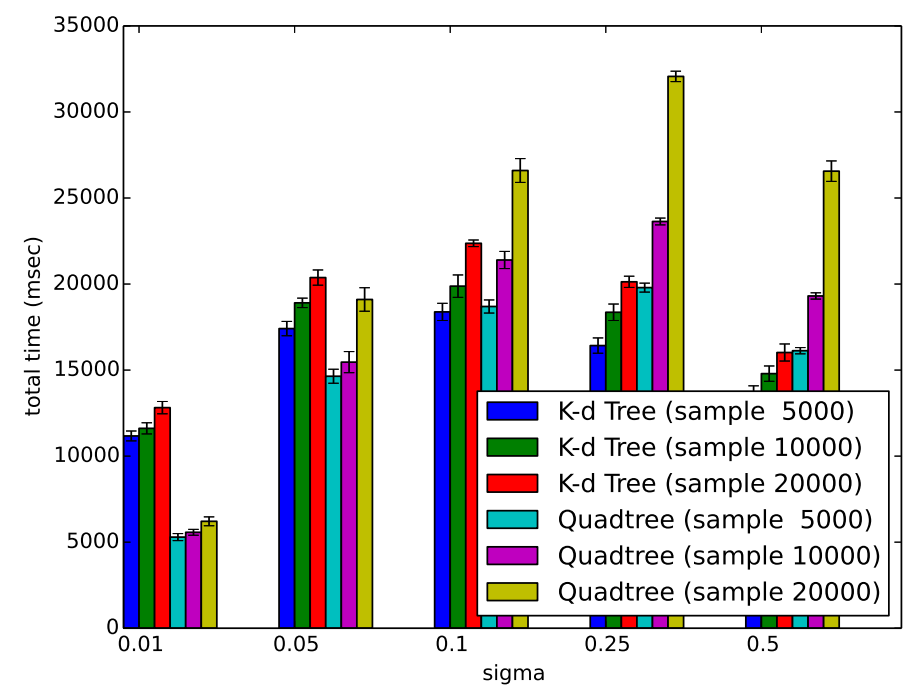

Figure 4.25: Total Time (3d, pts=10000)

part because the number of child nodes of a quadtree node is $2^{d}$ for a $d$-dimensional quadtree.

The kd-tree based implementation does not perform well in higher dimensional spaces either so the quadtree remains a viable implementation choice over the dimensions for which the odds-on tree exhibits good performance.

Cache hit rate measures the number of times the odds-on tree is able to answer a search without making use of the backup kd-tree structure. In general, the quadtree has a better hit rate than the kd-tree based odds-on tree. Typical results are shown in figure 4.27 .

For a node to be marked terminal the area it covers must have the same nearest neighbour. Since the interference queries are run from the corners of the node, a certain area outside of the node must also have the same nearest neighbour. A square node requires less area outside of the node to have the same nearest neighbour and so is more likely to be marked terminal. The area that must have the same nearest neighbour for a square and for a rectangular node is illustrated in figure 4.28.

A quadtree node always has equal length sides, but a kd-tree node is built by 


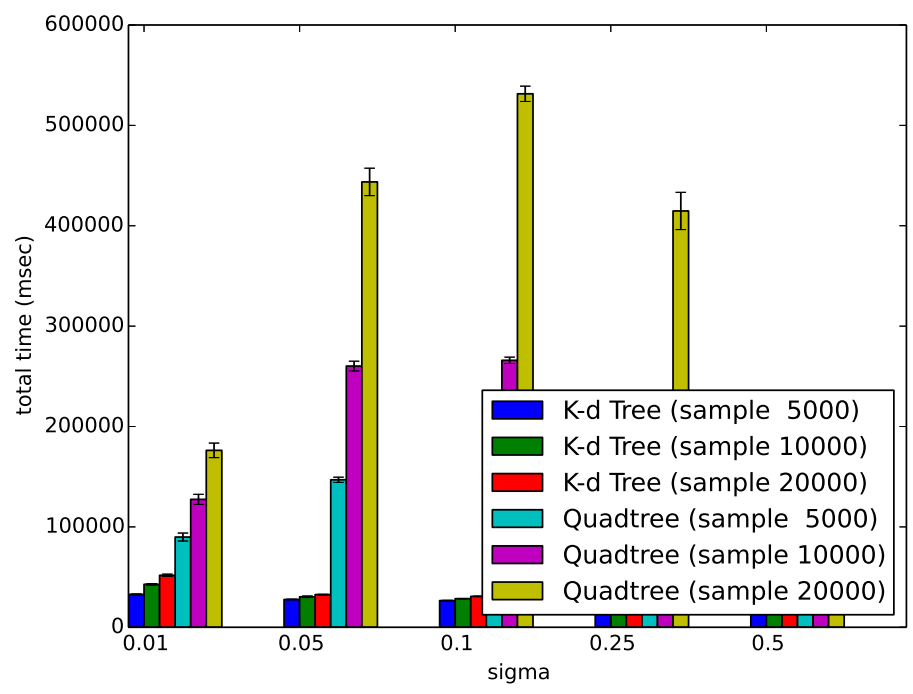

Figure 4.26: Total Time (8d, pts=10000)

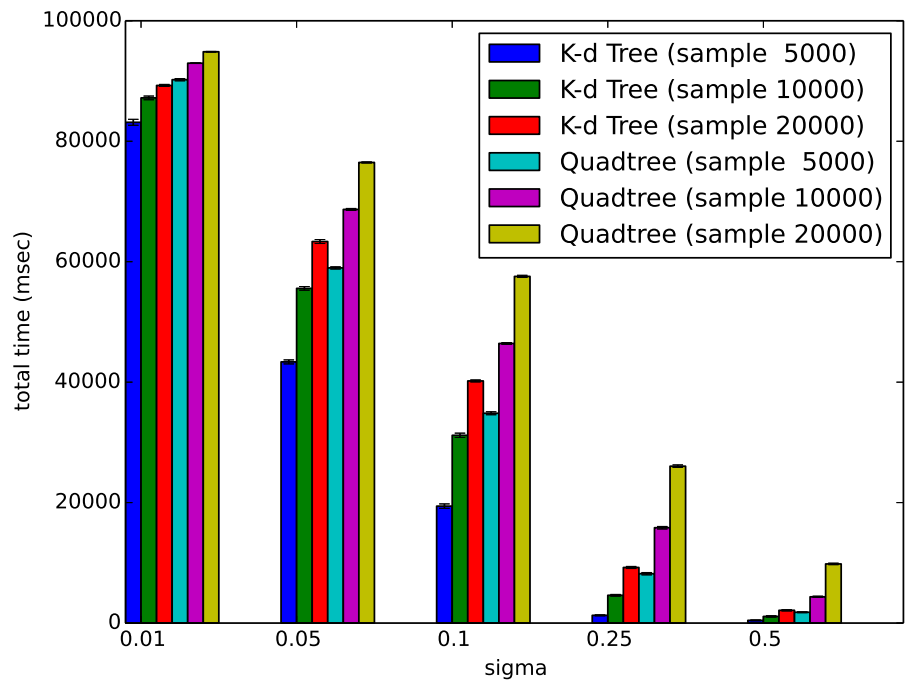

Figure 4.27: Cache Hits (2d, pts=10000) 


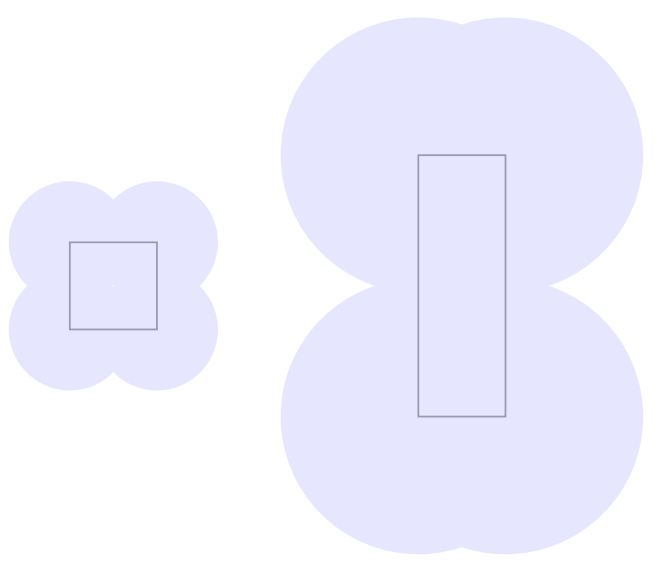

Figure 4.28: Square vs Rectangular Nodes

sub-dividing points and may have some sides much longer than others. Because of this, given a quadtree node and a kd-tree node of the same area the kd-tree node is less likely to be marked terminal, which means that a kd-tree based odds-on tree implementation is likely to have a greater number of smaller nodes, which will cause a lower cache hit rate.

The quadtree based implementation is able to build a more efficient cache than the kd-tree based implementation. Because of this, it exhibits better performance than the kd-tree based implementations for small values of search set sigma. 


\section{Chapter 5}

\section{Conclusions}

\section{$5.1 \quad$ Summary}

We have shown in this thesis that it is possible to create a practical data structure for biased neighbour search based upon odds-on trees. We developed implementations based upon quadtrees and kd-trees and through a series of careful experiments, compared their performance to our kd-tree implementation, on a number of synthetic datasets.

Our primary metric was running time but we also considered cache hit ratios, when comparing performance between odds-on tree implementations.

We found both implementations would outperform the kd-tree provided the entropy of the set of sites returned under the search distribution is sufficiently small. For small dimensions and sufficiently low search entropy, we found that the quadtree will outperform the kd-tree based implementation. For large dimensions, neither implementation performed bettered than a kd-tree.

Increasing the number of samples used to build the odds-on tree increased build time but resulted in building a better cache. Adjusting the maximum build depth did not have a clear effect on the build times or the quality of the cache. Increasing the point set size did not change the relative performance of the implementations. 


\subsection{Future Work}

The experimentation in this thesis focused on synthetic datasets due to the difficulty in finding "real world" datasets that included search distributions as well as the underlying point sets. Further experimentation should be done if suitable non-synthetic datasets are found.

The quadtree based implementation outperformed the kd-tree based implementation in some cases due to creating nodes that have equal side length. Our kd-tree implementation followed the original strategy in [9] of sorting the points into two equal sized groups. In applications of kd-trees, particularly in graphics, a number of other heuristic approaches have been developed that attempt to avoid creating nodes with a great disparity in side length [36]. Using these heuristics might provide better performance than either a quadtree or a standard kd-tree.

The Balanced Box Decomposition (BBD) Tree [4] is a more complicated data structure that maintains the equal space and equal partition size properties of quadtrees and kd-trees. It was not implemented as part of this thesis as its reported performance in the literature did not seem to justify dealing with the increased complexity of implementation. Given the results found that quadtrees build better caches than kd-trees it now seems worthwhile implementing.

This thesis did not investigate the k-nearest neighbour problem, where rather than returning a single nearest neighbour instead k nearest neighbours are returned. Intuitively, increasing $\mathrm{k}$ should have a negative impact on the cache as the area covered by a node that has the same $\mathrm{k}$ nearest neighbours will be smaller than the area covered by a node with only a single nearest neighbour. This could be investigated experimentally.

The odds-on tree data structure is based upon a cache containing a subset of points likely to returned as search results and a backup data structure containing all points.

Since the cache does not contain all points, returning multiple results requires searching both the cache and the backup data structure on every search. To be able to do so efficiently requires careful coordination between the two data structures and 
would never be as efficient as searching for a single point, which can potentially be answered using just the cache data structure.

The other alternative would be to construct the cache for all points which have the same predetermined number of nearest neighbours, which would result in each cache node covering a smaller area than in the single nearest neighbour search case. Again, this would not be as efficient as searching for a single point, and would require the (maximum) number of nearest neighbours to be returned to be fixed in advance.

A large portion of the time in doing a k-nearest neighbour search is spent in backtracking, where other potential nearest neighbours are investigated after the candidate nearest neighbours have been identified. The odds-on tree could potentially be used to prune the number of nodes investigated during back tracking, which might improve performance.

This thesis also did not investigate approximate nearest neighbour search. An approximate nearest neighbour search often dramatically reduces the amount of backtracking required to identify nearest neighbours and performs better in higher dimensional spaces.

Answering approximate nearest neighbour searches is easily done for a fixed approximation factor by creating a cache for that approximation factor. One could also perform experiments comparing exact search in an odds-on tree to approximate search in non-biased data structures. Both of these cases would provide for interesting experiments.

Approximate Voronoi Diagrams [26] were not considered for implementation in this thesis as they only work in the approximate case, but would be worthwhile investigating in the future if further work were done with approximate nearest neighbour search. 


\section{Bibliography}

[1] Aragon, C. R., Seidel, R. (1996) Randomized Search Trees. In Algorithmica, Vol. 16, Number 4/5, pp. $464-497$.

[2] Arya, S., Mount, D. M. (1993) Algorithms for Fast Vector Quantization, Proceedings of DCC '93, IEEE Press, pp $381-390$.

[3] Arya, S., Mount, D. M. (1993) Approximate nearest neighbour queries in Fixed Dimensions, Proceedings of the 4th ACIM-SIAM Symposium on Discrete Algorithms, pp $271-280$.

[4] Arya, S., Mount, D. M., Netanyahu, N. S., Silverman, R. Wu, A. Y. (1998) An Optimal Algorithm for Approximate Nearest Neighbor Searching in Fixed Dimensions, Journal of the ACM, Volume 45, Issue 6, pp 891 - 923.

[5] Arya, S., Malamatos, T. Mount, D. M. (2001) A Simple Entropy-Based Algorithm for Planar Point Location. In Proceedings of the Twelfth Annual ACM-SIAM Symposium on Discrete Algorithms, pp. 262 - 268.

[6] Arya, S., Malamatos, T. Mount, D. M. (2002) Space-efficient Approximate Voronoi Diagrams, Proceedings of the 33rd Annual ACM Symposium on the Theory of Computing, pp. $721-730$.

[7] Bagchi, A. Buchsbaum, A. L., Goodrich, M. T. (2005) Biased Skip Lists. In Algorithmica Vol. 42, pp. $31-48$.

[8] Bent, S. W., Sleator, D. D., Tarjan, R. E. (1984) Biased Search Trees, In SIAM Journal on Computing, Volume 14, pp. $545-568$. 
[9] Bentley, J. L. (1975) Multidimensional Binary Search Trees Used for Associative Searching, In Communications of the ACM, Volume 18, Number 9, pp. $509-517$.

[10] Bentley, J. L. (1980) Multidimensional Divide-and-Conquer. In Communications of the ACM, Volume 23, pp. $214-229$.

[11] Bern, M., Eppstein, D., Teng, S-H. (1993) Parallel Construction of Quadtrees and Quality Triangulations, WADS '93: Proceedings of the Third Workshop on Algorithms and Data Structures, pp. 188 - 199.

[12] Bose, P., Devroye, L, Douieb, K., Dujmovic, V., King, J., Morin, P. (2010) Odds-On Trees, preprint, retrieved from http://arxiv.org/abs/1002.1092 on April $27,2013$.

[13] Chan, T. M. (2006) A Minimalist's Implementation of an Approximate Nearest Neighbour Algorithm in Fixed Dimensions, unpublished note, retrieved from http://www.cs.uwaterloo.ca/ tmchan/pub\_ann.html on July 29, 2012.

[14] Chazelle, B. (1993) Cutting hyperplanes for divide-and-conquer. Discrete and Computational Geometry 9, pp. 145 - 158.

[15] Clarkson, Kenneth L. (1983) Fast Algorithms for the All Nearest Neighbors Problem, FOCS '83: Proceedings of the Twenty-fourth Symposium on Foundations of Computer Science, Tucson, AZ.

[16] Connor, M. and Kumar, P. (2009) Fast Construction of k-Nearest Neighbor Graphs For Point Clouds, IEEE Transactions of Visualization and Computer Graphics, September 2009.

[17] de Berg., M. van Kreveld, M., Overmars, M., Schwarzkopf, O. (2000) Computational Geometry: Algorithms and Applications, 2nd Edition, Springer-Verlag, Berlin Heidelberg New York.

[18] Dujmovic, V., Howat, J., Morin, P. (2009) Biased Range Trees In Proceedings of the twentieth Annual ACM-SIAM Symposium on Discrete Algorithms, New York, New York, pp. $486-495$. 
[19] Edelsbrunner, H., Guibas, L. J., Stolfi, J. (1986) Optimal Point Location in a Monotone Subdivision, SIAM Journal on Computing, 15(2), pp. 317 - 340.

[20] Eppstein, D., Goodrich, M. T., Sun, J. Z. (2008) The Skip Quadtree: A Simple Dynamic Data Structure for Multidimensional Data, Int. Journal on Computational Geometry and Applications, 18(1/2), pp. $131-160$.

[21] Ergun, F., Sahinalp, S. C., Sharp, J., Sinha, R. K. (2001) Biased Skip Lists for Highly Skewed Access Patterns, Proceedings of ALENEX 2001, Lecture Notes in Computer Science, Volume 2153, pp. 216 - 229.

[22] Feigenbaum, J., Tarjan, R. E. (1983) Two New Kinds of Biased Search Tree, In Bell Systems Technical Journal, Vol. 62, No. 10, pp. 3139 - 3158.

[23] Finkel, R. A. and Bentley, J. L. (1974) Quad Trees A Data Structure for Retrieval on Composite Keys, Acta Informatica, 4(1), pp. 1 - 9.

[24] Friedman, J. H., Bentley, J. L., Finkel, R. A. (1977) An Algorithm for Finding Best Matches in Logarithmic Expected Time, ACM Transactions on Mathematical Software, Vol. 3 Number 3, pp. 209 - 226.

[25] Guttman, A. (1984) R-Trees: A Dynamic Index Structure for Spatial Searching, SIGMOD '84: Proceedings of the 1984 ACM SIGMOD International Conference on the Management of Data, pp. $47-58$.

[26] Har-Peled, S. (2001) A Replacement for Voronoi Diagrams of Near Linear Size, FOCS '01: Proceedings of the Symposium on Foundations of Computer Science, pp. $94-103$.

[27] Iacono, J. (2001) Optimal Planar Point Location In Proceedings of the Twelfth Annual ACM-SIAM Symposium on Discrete Algorithms, pp. 240 - 241.

[28] Indyk, P., Motwani, R. (1998) Approximate Nearest Neighbors: Towards Removing the Curse of Dimensionality, In Proceedings of the Thirtieth Annual ACM Symposium on Theory of Computing, pp. $604-613$. 
[29] Kirkpatrick, D. (1983) Optimal Search in Planar Subdivisions, Siam Journal of Computing, Vol. 12, No. 1, pp. $28-34$.

[30] Knuth, D. E. (1998) The Art of Computer Programming, Second Edition, Volume 3, Sorting and Searching. Addison-Wesley, Reading, Massachusetts, USA.

[31] Liu, T., Moore, A. W., Gray, A., Yang, K. (2004) An Investigation of Practical Approximate Nearest-Neighbour Algorithms, Proceedings of Neural Information Processing Systems(NIPS 2004), pp. 825 - 832.

[32] Mehlhorn, K. (1975) Nearly Optimal Binary Search Trees In Acta Informatica, $5(1)$, pp. $287-295$.

[33] Mines, M. A., Hanson, B. F., Shoup, J. E. (1978) Frequency of Occurence of Phonemes in Conversational English, Language and Speech, vol. 21 no. 3, pp. 221 $-241$

[34] Morton, G. M. (1966), A Computer Oriented Geodetic Data Base; and a New Technique in File Sequencing, Technical Report, IBM Ltd., Ottawa, Canada.

[35] Mount, D. M., Arya, S. (2012) ANN: A Library for Approximate Nearest Neighbor Searching, retrieved from http://www.cs.umd.edu/ mount/ANN on July 29, 2012.

[36] Pharr, M., Humphreys, G. (2010) Physically Based Rendering MorganKaufmann, San Francisco, CA.

[37] Pugh, W. (1990) Skip Lists: A Probabilistic Alternative to Balanced Trees. In Communications of the ACM, 33(6), pp. $668-676$.

[38] Rudin, W. (1976) Principles of Mathematical Analysis, Third Edition McGrawHill, New York, NY.

[39] Samet, H. (2006) Foundations of Multidimensional and Metric Data Structures Morgan-Kaufmann, San Francisco, CA. 
[40] Sarnak, N. and Tarjan, R. E. (1986) Planar Point Location Using Persistent Search Trees. Communications of the ACM, 29(7), pp. $669-679$.

[41] Shewchuk, J. R. (2013) Lecture Notes on Geometric Robustness, Retrieved from http://www.cs.berkeley.edu/ jrs/meshpapers/robnotes.pdf on October 13, 2014.

[42] Shannon, Claude E. (1948) A Mathematical Theory of Communication, Bell System Technical Journal, 27, pp. $379-423$ and $623-656$.

[43] Sleator, D. D., Tarjan, R. E. (1985) Self-adjusting binary search trees. In Journal of the ACM, 32, pp. $652-686$.

[44] Tambovtsev, Y. and Martindale, C. (2007) Phoneme Frequencies Follow a Yule Distribution. SKASE Journal of Theoretical Linguistics 4: 2.

[45] Williams, H. E., Zobel, J., Heinz, S. (2001) Self-Adjusting Trees in Practice for Large Text Collections, In Software - Practice and Experience, Volume 31, pp. $925-939$. 